\title{
HIGHER-ORDER SYNCHRONIZATION FOR A DATA ASSIMILATION ALGORITHM FOR THE 2D NAVIER-STOKES EQUATIONS
}

\author{
ANIMIKH BISWAS ${ }^{1}$ AND VINCENT R. MARTINEZ ${ }^{2, \dagger}$
}

\begin{abstract}
We consider the two-dimensional (2D) Navier-Stokes equations (NSE) with space periodic boundary conditions and an algorithm for continuous data assimilation developed by Azouani, Olson and Titi (2014). The algorithm is based on the observation that existence of finite determining parameters for nonlinear dissipative systems can be exploited as a feedback control mechanism for a companion system into which observables, e.g, modes, nodes, or volume elements, are input directly for the purpose of assimilation. It has been shown that in the case of the 2D NSE, the approximating solution induced by the algorithm synchronizes with the exact solution of the 2D NSE in the topology of the Sobolev space, $H^{1}$, provided that the number of observed modes, nodes, or volume elements is sufficiently large in terms of the Grashof number. In this article, we adapt a technique, introduced by Grujić and Kukavica (1998) and Kukavica (1999) to obtain good estimates of the analyticity radius for the 2D NSE, and show that one can in fact obtain synchronization in the analytic Gevrey class in the case of modal observables, given sufficiently many, but fixed number of such observables. For these types observables, we additionally show that synchronization in the uniform norm, $L^{\infty}$, can be achieved by assuming the same number of modal observables (in terms of the order of the Grashof number) as is required for the $H^{1}$ synchronization.
\end{abstract}

Keywords: 2D Navier-Stokes equations, higher-order synchronization, Data assimilation, nudging scheme

\section{INTRODUCTION}

In 1904, physicist and meteorologist V. Bjerknes defined "the problem of prognosis as nothing less than the integration of the equations of motion of the atmosphere" (cf. [1, 2]). To do so, however, one must initialize the equations with the exact state of the system. On the other hand, the launch of the first weather satellites in the 1960s, has allowed for weather data to be collected nearly continuously in time. The question, then, is how can one use the coarse observational data to obtain an accurate representation of the current state of the system? This is the main goal of continuous data assimilation.

Recently, a new algorithm for continuous data assimilation was proposed and studied in the case of the 2D NSE by Azouani, Olson, and Titi [3], in which the observed data is inserted directly into the prognostic equations as an additional term in such a way that it serves to relax the solution towards the observations. By now, this algorithm has been studied for several important physical models including the 1D Chaffee-Infante equation, 2D Boussinesq, 3D Brinkman-Forchheimer extended Darcy equations, 3D Bénard convection in porous media, and

Date: July 13, 2016.

$\dagger$ denotes corresponding author. 
3D NS $\alpha$-model, (cf. $[4,5,6,7,8,9]$ ), as well as the case of noisy data [10]. More recently, the algorithm has been adapted as a discrete data assimilation algorithm (cf. [11]).

We will describe this algorithm for the equation of interest in this paper, namely the 2D incompressible Navier-Stokes equations (NSE) on $\Omega=[-L / 2, L / 2]^{2}$ :

$$
\partial_{t} u-\nu \Delta u+u \cdot \nabla u+\nabla p=f, \quad \nabla \cdot u=0, \quad u(x, 0)=u_{0}(x)
$$

where $u$ is the velocity vector field, $p$ the scalar pressure, $f$ an external forcing, $u_{0}$ the initial velocity, and $\nu$ is the kinematic viscosity. In practice, one does not know the exact state, $u_{0}$, of the system, and one must choose a function with which to initialize the system. The algorithm of Azouani, Olson, and Titi was inspired from ideas in control theory and based on a feedback control paradigm. In particular, in the absence of measurement noise, let $I_{h}(u)$ represent the collected observables, where $u$ corresponds to a reference solution satisfying (1.1) with either periodic or no-slip Dirichlet boundary conditions. Then depending on the boundary conditions satisfied by $u$, define an approximating solution, $v$, of $u$ that satisfies the following equation with either periodic or no-slip Dirichlet boundary conditions over $\Omega$ :

$$
\partial_{t} v-\nu \Delta v+v \cdot \nabla v+\nabla q=f-\mu I_{h}(v-u), \quad \nabla \cdot v=0, \quad v(x, 0)=v_{0}(x),
$$

where $\mu$ is an appropriately chosen tuning parameter and the initial value, $v_{0}$, can be chosen arbitrary (e.g. $\left.v_{0} \equiv 0\right)$. We will refer to (1.2) as the Azouani-Olson-Titi data assimilation system. It is shown in [3] that $v$ synchronizes with $u$ exponentially as time tends to infinity. The algorithm would then be to input observed data into (1.2) and integrate (1.2) forward in time to produce an approximating solution, $v$, with which one can then use to initialize (1.1).

One may view $I_{h}$ as an interpolant operator, which is linear and of finite rank, and moreover $I_{h}(u)$ can be constructed from the observed data. Examples of such operators include projection onto a grid of nodal values or local averages of mesh size $h$, as well as projection onto finitely many modal observables. For the algorithm, however, one only needs to assume that $I_{h}$ is linear, of finite-rank, and satisfies certain approximation-of-identity properties. In this article, we will consider the case when $I_{h}$ is given by projection onto finitely many Fourier modes.

The success of the algorithm relies on the property that the 2D NSE exhibits finitely many "determining parameters". That is, knowledge of sufficiently many, for example, nodal values for all times, determines all other nodal values asymptotically, as time tends to infinity. This property is precisely what guarantees that the approximating solution, $v$, will synchronize with the reference solution, $u$, from which the observed data is assumed to be derived. One is referred to a seminal paper of Foias and Prodi [12] for the existence of determining parameters in the modal case, and $[13,14,15,16]$ for the nodal and volume elements case.

It was shown in [3] that the approximating solution, $v$, satisfying (1.2) synchronizes in the topology of the $H^{1}$-norm exponentially in time, provided that $\mu$ is chosen sufficiently large and that the spatial resolution, $h$, of the observables satisfies $h \lesssim \kappa_{0}^{-1} \mathcal{G}^{-\frac{1}{2}}(1+\log (1+\mathcal{G}))^{-1 / 2}$, in the periodic case, or $h \lesssim \kappa_{0}^{-1} \mathcal{G}$ in the no-slip case, where $\mathcal{G}$ denotes the Grashof number and is defined by

$$
\mathcal{G}:=\frac{1}{\nu^{2} \kappa_{0}^{2}} \sup _{t>0}\|f(t)\|_{L^{2}}, \quad \kappa_{0}=2 \pi / L .
$$

From the point of view of the Sobolev embedding theorem, it is thus natural to ask under what conditions can one ensure synchronization in the topology of uniform convergence, that 
is, with respect to the $L^{\infty}$-norm. In this paper, we show that in case of the periodic boundary conditions with $I_{h}$ given by an appropriate modal projection, the solution to the AzouaniOlson-Titi system (1.2) does indeed synchronize with the reference solution in the $L^{\infty}$-topology under essentially the same number of modal observables that ensure synchronization in the $H^{1}$ topology. In particular, we have the following theorem. where $\Omega=[-L / 2, L / 2]^{2}$ and we impose space periodic boundary conditions on (1.1), (1.2).

Theorem 1. Let $p \in[2, \infty)$ be an even integer and let $f \in L^{\infty}\left((0, \infty), L^{2}(\Omega)^{2}\right)$ be such that $\nabla \times f \in L^{\infty}\left((0, \infty), L^{p}(\Omega)\right)$ and $f$ has mean-zero. Let $u_{0}, v_{0} \in H^{1}(\Omega)^{2}$ be divergence-free and mean-zero. Suppose that $\nabla \times v_{0} \in L^{p}(\Omega)$. There exist absolute constants $c_{0}, c_{1}$, with $c_{0}=c_{0}(p)$, such that if $m, \mu$ satisfy

$$
2^{2 m} \frac{\nu \kappa_{0}^{2}}{\mu} \geq c_{0}(p) \quad \text { and } \quad \mu \geq c_{1} \nu \kappa_{0}^{2} \mathcal{G}(1+\log (1+\mathcal{G}))
$$

then

$$
\|\nabla \times v(t)-\nabla \times u(t)\|_{L^{p}} \rightarrow 0 \quad \text { exponentially as } t \rightarrow \infty,
$$

where $u, v$ are the corresponding strong solutions of (1.1), (1.2), respectively, with $I_{h}=P_{2^{m}}$, where $P_{N}$ denotes projection onto the Fourier modes with wave-numbers $\left|k_{1}\right|,\left|k_{2}\right| \leq N$.

By interpolation, we may then immediately deduce the following.

Corollary 1.1. Under the hypotheses of Theorem 1, with p sufficiently large, we have

$$
\|v(t)-u(t)\|_{L^{\infty}} \rightarrow 0 \text { exponentially as } t \rightarrow \infty \text {. }
$$

Proof. Let $w(t):=v(t)-u(t)$. Since $w$ is divergence-free, we have (cf. [17])

$$
\|\nabla w\|_{L^{p}} \leq C\|\nabla \times w\|_{L^{p}}, \quad 1<p<\infty .
$$

On the other hand, by the Sobolev embedding theorem and Biot-Savart law, we have

$$
\|w\|_{L^{p}} \leq C\|\nabla w\|_{L^{2}}=\|\nabla \times w\|_{L^{2}}, \quad 1<p<\infty .
$$

We observe that $\|\nabla \times w\|_{L^{2}}$ is uniformly bounded by Proposition 2.3 (in fact, exponentially decaying to 0 as $t \rightarrow \infty)$. Since $w$ has mean zero over $\Omega$, by the Gagliardo-Nirenberg interpolation inequality, we have

$$
\|w(t)\|_{L^{\infty}} \leq C\|\nabla w(t)\|_{L^{p}}^{2 / p}\|w(t)\|_{L^{p}}^{1-2 / p}
$$

for $p>2$. From Theorem 1, we may conclude that $\|w(t)\|_{L^{\infty}} \rightarrow 0$ exponentially as $t \rightarrow \infty$.

We prove Theorem 1 in Section 3 by performing the a priori estimates in $L^{p}$ for (1.2), but at the level of the "vorticity," $\eta=\nabla \times v$. By exploiting the transport structure of the equation for $\eta$, one can then interpolate with the result of Azouani, Olson, and Titi (Theorem 2.3) to guarantee the synchronization property. To obtain the proper $L^{p}$ estimates for $\eta$, we make use of smooth modal projections as given by the Littlewood-Paley projections (see (2.21)), as opposed to the "rough" modal projections defined by (2.24) (see also (2.26)).

Since modal observables retain information regarding all higher-order derivatives of the reference solution, one can in fact ensure synchronization in a far stronger topology, i.e., with respect to the analytic Gevrey norm (see (5.2)), provided that the the number of known observables 
is sufficiently high. First, let $f$ be the restriction of an analytic function $f(x, y, t)+i g(x, y, t)$ $x, y \in \Omega$ with uniform analyticity radius $\delta_{f}>0$. We define

$$
\begin{aligned}
M_{f} & :=\sup _{t \in[0, \infty),|y| \leq \delta_{f}}\|f(\cdot, y, t)\|_{L^{\infty}(\Omega)}+\|g(\cdot, y, t)\|_{L^{\infty}(\Omega)}, \\
M_{F} & :=\sup _{t \in[0, \infty),|y| \leq \delta_{f}}\|F(\cdot, y, t)\|_{L^{\infty}(\Omega)}+\|G(\cdot, y, t)\|_{L^{\infty}(\Omega)},
\end{aligned}
$$

where $F:=\nabla \times f$ and $G:=\nabla \times g$. We also define the following modified Grashof numbers:

$$
\mathcal{G}_{\infty}:=\frac{1}{\nu^{2} \kappa_{0}^{3}} \sup _{t>0}\|f(t)\|_{L^{\infty}} \text { and } \quad \widetilde{\mathcal{G}}_{\infty}:=\frac{1}{\nu^{2} \kappa_{0}^{4}} \sup _{t>0}\|F(t)\|_{L^{\infty}} .
$$

Theorem 2. Let $f \in L^{\infty}\left((0, \infty) ; L^{\infty}(\Omega)^{2}\right)$ be the restriction of an analytic function $f(x, y, t)+$ $i g(x, y, t)$ such that for some $\delta_{f}>0, f$ satisfies $M_{f}, M_{F}<\infty$. Let $u_{0}, v_{0}$ be divergence-free and mean-zero such that $u_{0} \in H^{1}(\Omega)^{2}$ and $\nabla \times v_{0} \in L^{\infty}(\Omega)$. There exists $\mu>0$ sufficiently large, $\delta_{*}>0$ sufficiently small, and absolute constants $c_{0}, c_{1}>0$ such that if $v_{0}$ and $m$ satisfy

$$
2^{m} \geq c_{1} \frac{\mu}{\nu \kappa_{0}^{2}}\left(1+\log _{+} \widetilde{\mathcal{G}}_{\infty}\right)^{2}
$$

then for all $\gamma<\delta_{*}$, we have $\left\|e^{\gamma A^{1 / 2}}(v(t)-u(t))\right\|_{H^{1}(\Omega)} \rightarrow 0$ exponentially as $t \rightarrow \infty$, where $A$ denotes the Stokes operator.

Remark 2.1. We note that one may have to pass through a transient time before the exponential decay rate comes into effect (see, for instance, Lemma 3.2.1 and Proposition 4.1).

Also, the case where $f$ is time-independent and has only finitely many modes, i.e., $P_{N} f=f$ for some $N>0$, then for $\mathcal{G} \geq 1$, we require $\mu$ to satisfy

$$
\mu \geq C \nu \kappa_{0}^{2} \mathcal{G}^{5 / 2}\left(1+\log _{+} \mathcal{G}\right)^{1 / 4}
$$

for some absolute constant $C>0$ sufficiently large. It follows that we require the number of modes $m$ to satisfy

$$
2^{m} \geq C \mathcal{G}^{5 / 2}\left(1+\log _{+} \mathcal{G}\right)^{13 / 4}
$$

We refer to (5.5) in the proof of Theorem 2 and Remark 4.1.1 to obtain (1.8).

Remark 2.2. In [18], uniform convergence was in fact observed in the numerical experiments performed in [19]. It was also observed in [19] that much less modes were required to see the exponential decay than the analytical estimate given by (1.4) suggested. It would be interesting to see if one can observe the convergence in the Gevrey norm using far fewer modes than suggested by (1.7).

To prove this, we first establish that the approximating solution, $v$, is real-analytic over $\Omega$ and obtain a lower bound estimate for its uniform spatial radius of analyticity for $t$ sufficiently large. We do this in Section 4 by adapting a technique of Kukavica [20] (see also [34]), which was originally applied to obtain the best-to-date lower bounds on the radius of analyticity for the 2D and 3D NSE. This, in turn, implies uniform-in-time bounds on the Gevrey norm of $v$, with which we will interpolate to obtain the corresponding synchronization property. We again emphasize the role of the smooth modal projections, which make useful harmonic analysis tools available (see Section 2.3) that ultimately allow us to adapt the technique of Kukavica to the data assimilation system. 


\section{Preliminaries}

By rescaling variables, we may assume that $\Omega=\mathbb{T}^{2}$, where $\mathbb{T}^{2}=\mathbb{R}^{2} /(2 \pi \mathbb{Z})^{2}$ and $\nu=1$. Indeed, for $\kappa_{0}:=2 \pi / L$, we may set

$$
\bar{x}=\kappa_{0} x, \quad \bar{t}=\nu \kappa_{0}^{2} t, \quad \bar{u}=\left(\nu \kappa_{0}\right)^{-1} u, \quad \bar{p}=\left(\nu \kappa_{0}\right)^{-2} p, \quad \bar{f}=\left(\nu^{-2} \kappa_{0}^{-3}\right) f .
$$

Then (1.1) becomes

$$
\partial_{\bar{t}} \bar{u}-\Delta_{\bar{x}} \bar{u}+\bar{u} \cdot \nabla_{\bar{x}} \bar{u}+\nabla_{\bar{x}} \bar{p}=\bar{f}, \quad \nabla_{\bar{x}} \cdot \bar{u}=0, \quad \bar{u}(\bar{x}, 0)=\bar{u}_{0}(\bar{x}),
$$

subject to periodic boundary conditions. As with (2.2), we will also work in a non-dimensionalized setting for the data assimilation system (2.3). In particular, we will consider the equation

$$
\partial_{t} \bar{v}-\Delta_{\bar{x}} \bar{v}+\bar{v} \cdot \nabla_{\bar{x}} \bar{v}+\nabla_{\bar{x}} \bar{q}=\bar{f}-\bar{\mu} P_{N}(\bar{v}-\bar{u}), \quad \nabla_{\bar{x}} \bar{v}=0, \quad \bar{v}(\bar{x}, 0)=\bar{v}_{0}(\bar{x}),
$$

subject to periodic boundary conditions, where $\bar{\mu}=\mu /\left(\nu \kappa_{0}^{2}\right), P_{N}$ denotes orthogonal projection onto wave-numbers $\leq N$ (see $(2.24))$

We will also consider the vorticity forms of (2.2) and (2.3). The vorticity form of (2.2) is given by

$$
\partial_{\bar{t}} \bar{\omega}-\Delta_{\bar{x}} \bar{\omega}+\bar{u} \cdot \nabla_{\bar{x}} \bar{\omega}=\nabla_{\bar{x}} \times \bar{f}, \quad \nabla_{\bar{x}} \cdot \bar{u}=0, \quad \omega=\nabla_{\bar{x}} \times \bar{u}, \quad \bar{\omega}(\bar{x}, 0)=\nabla \times \bar{u}_{0}(\bar{x}),
$$

where $\bar{f}$ is the forcing term of the velocity equation, (2.2). Similarly, the vorticity form of (2.3) is given by

$\partial_{\bar{t}} \bar{\eta}-\Delta_{\bar{x}} \bar{\eta}+v \cdot \nabla \bar{\eta}=\nabla_{\bar{x}} \times \bar{f}-\mu P_{N}(\bar{\eta}-\bar{\omega}), \quad \nabla_{\bar{x}} \cdot \bar{v}=0, \quad \bar{\eta}=\nabla_{\bar{x}} \times \bar{v}, \quad \bar{\eta}(\bar{x}, 0)=\nabla \times \bar{v}_{0}(\bar{x})$.

It will also be useful to view (2.2) as an evolution equation over a Hilbert space $H$ (see (2.9)), which is a closed subspace of mean-zero, divergence-free vector fields in $\left(L^{2}\left(\mathbb{T}^{2}\right)\right)^{2}$. We describe this functional setting more precisely in Section 2.1. The functional form of (2.2) is given by

$$
\frac{d u}{d t}+A u+B(u, u)=\mathcal{P} f, \quad u(0)=u_{0},
$$

where $A=-\mathcal{P} \Delta$ denotes the Stokes operator with eigenvalues $|k|^{2}$, for $k \in \mathbb{Z}^{2} \backslash\{\mathbf{0}\}$, and $\mathcal{P}$ is the Leray projection onto divergence-free vector fields, i.e., $\mathcal{P}:\left(L^{2}\left(\mathbb{T}^{2}\right)\right)^{2} \rightarrow\left(L_{\sigma}^{2}\left(\mathbb{T}^{2}\right)\right)^{2}$ whose Fourier transform is determined by

$$
\widehat{\mathcal{P} u}(\mathbf{0})=\hat{u}(\mathbf{0}), \quad \widehat{\mathcal{P} u}(k):=\hat{u}(k)-(k \cdot \hat{u}(k)) \frac{k}{|k|^{2}}, \quad k \in \mathbb{Z}^{2} \backslash\{\mathbf{0}\} .
$$

In particular, the Fourier symbol of $\mathcal{P}$ is given by $\operatorname{Id}_{2 \times 2}-(\xi \otimes \xi) /|\xi|^{2}$, so that

$$
\|\mathcal{P} u\|_{L^{p}} \leq c_{2.7}\|u\|_{L^{p}}, \quad 1<p<\infty .
$$

for some absolute constant $c_{2.7}=c_{2.7}(p)$ (cf. [21, 22]).

Similarly, we will also view (2.3) in the same functional setting as (2.6):

$$
\frac{d v}{d t}+A v+B(v, v)=\mathcal{P} f-\mu P_{2^{m}}(v-u), \quad v(0)=v_{0} \in V
$$

From now on, we will work in the non-dimensionalized setting determined by (2.1). In particular, henceforth, $\Omega=\mathbb{T}^{2}$. Let us also drop the bar notation for the rest of the article. 
2.1. Functional setting. Let $\mathcal{V}$ denote the space of two-dimensional divergence-free vector fields over $\mathbb{T}^{2}$ with mean zero, so that

$$
H:=\overline{\mathcal{V}}^{L^{2}\left(\mathbb{T}^{2}\right)} \quad \text { and } \quad V:=\overline{\mathcal{V}}^{H^{1}\left(\mathbb{T}^{2}\right)},
$$

where $H^{1}\left(\mathbb{T}^{2}\right)$ is the subspace of $L^{2}\left(\mathbb{T}^{2}\right)$ whose first weak derivatives also belong to $L^{2}\left(\mathbb{T}^{2}\right)$. Then $H$ and $V$ are Hilbert spaces with inner products

$$
(u, v):=\int_{\mathbb{T}^{2}} u(x) \cdot v(x) d x \quad \text { and } \quad((u, v)):=\sum_{i, j=1}^{2} \int_{\mathbb{T}^{2}} \partial_{x_{j}} u_{i} \partial_{x_{j}} v_{i} d x
$$

respectively, which induce the following norms:

$$
\|u\|_{H}:=\|u\|_{L^{2}}, \quad\|u\|_{V}:=\left\|A^{1 / 2} u\right\|_{L^{2}} .
$$

We denote the domain of $A^{\alpha / 2}$ by $=\mathcal{D}\left(A^{\alpha / 2}\right)=\left\{u \in V: A^{\alpha / 2} u \in H\right\}$, which is equipped with the norm

$$
\|u\|_{\mathcal{D}\left(A^{\alpha / 2}\right)}:=\left\|A^{\alpha / 2} u\right\|_{L^{2}}
$$

Observe that for divergence-free vector fields, $u$, periodic with respect to $\mathbb{T}^{2}$, we have

$$
A u=-\mathcal{P} \Delta u=-\Delta \mathcal{P} u=-\Delta u .
$$

Recall that one has the Poincaré inequalities:

$$
\|u\|_{H} \leq\|u\|_{V} \leq\|u\|_{\mathcal{D}\left(A^{\alpha / 2}\right)}, \quad \alpha \geq 1 .
$$

We will also make use of the following orthogonality property:

$$
\left(v \cdot \nabla \phi, \phi^{p-1}\right)=0,
$$

for smooth, two-dimensional vector fields, $v$, periodic over $\mathbb{T}^{2}$, satisfying $\nabla \cdot v=0$ and smooth scalar functions, $\phi$, periodic over $\mathbb{T}^{2}$ with $p \geq 2$. It can be proved by performing an integration by parts.

2.2. Calculus inequalites. We will make use of the following interpolation inequalities. Recall that the fractional Laplacian, $(-\Delta)^{\alpha / 2}$, can be defined by its Fourier symbol, $|\xi|^{\alpha}$.

Lemma 2.2.1. Let $0 \leq \alpha<\beta$ and $d \geq 1$. Suppose $g$ is periodic in $\mathbb{T}^{d}$. There exists an absolute constant $C>0$ such that

$$
\left\|(-\Delta)^{\alpha / 2} g\right\|_{L^{2}} \leq C\left\|(-\Delta)^{\beta / 2} g\right\|_{L^{2}}^{\frac{\alpha}{\beta}}\|g\|_{L^{2}}^{1-\frac{\alpha}{\beta}}
$$

Let us also recall the Sobolev embedding for convenience.

Lemma 2.2.2. Let $1 \leq q<p<\infty$ and $d \geq 1$. Suppose that $\alpha q<d$ and $1 / p=1 / q-\alpha / d$. Suppose $g$ is periodic in $\mathbb{T}^{d}$ and has mean zero. There exists an absolute constant $C>0$ such that

$$
\|g\|_{L^{p}} \leq C\left\|(-\Delta)^{\alpha / 2} g\right\|_{L^{q}}
$$

For a periodic function $g$ in $\mathbb{T}^{d}$, the Wiener algebra norm is defined by $\|g\|_{\mathcal{W}}=\sum_{k \in \mathbb{Z}^{d}}|\hat{g}(k)|$. Recall that $\|g\|_{L^{\infty}} \lesssim\|g\|_{\mathcal{W}}$. Two important borderline cases of the Sobolev inequalities given below are the Agmon's inequality and the Brezis-Gallouet inequality. 
Lemma 2.2.3 (Agmon's inequality). Suppose $g$ is periodic in $\mathbb{T}^{2}$. There exists an absolute constant $C>0$ such that

$$
\|g\|_{\mathcal{W}} \leq C\|\Delta g\|_{L^{2}}^{1 / 2}\|g\|_{L^{2}}^{1 / 2}
$$

We also have the following inequality.

Lemma 2.2.4 (Brezis-Gallouet inequality). Suppose $g$ is periodic in $\mathbb{T}^{2}$. There exists an absolute constant $C>0$ such that

$$
\|g\|_{\mathcal{W}} \leq C\left\|(-\Delta)^{1 / 2} g\right\|_{L^{2}}\left(1+\log \left(\frac{\|\Delta g\|_{L^{2}}^{2}}{\left\|(-\Delta)^{1 / 2} g\right\|_{L^{2}}^{2}}\right)\right)^{1 / 2} .
$$

We will also make use of the following inequality (cf. [23, 24]).

Lemma 2.2.5. Let $\alpha>0, d \geq 1$. Suppose that $g, h$ are periodic in $\mathbb{T}^{d}$ and have mean zero. Then

$$
\left\|(-\Delta)^{\alpha / 2}(g h)\right\|_{L^{p}} \leq C\left(\left\|(-\Delta)^{\alpha / 2} g\right\|_{L^{p_{1}}}\|h\|_{L^{p_{2}}}+\|g\|_{L^{p_{3}}}\left\|(-\Delta)^{\alpha / 2} h\right\|_{L^{p_{4}}}\right),
$$

where $1 / p=1 / p_{1}+1 / p_{2}=1 / p_{3}+1 / p_{4}$ and $1<p, p_{1}, p_{2}, p_{3}, p_{4}<\infty$.

2.3. Littlewood-Paley decomposition and related inequalities. We note that we will state define the decomposition over $\mathbb{R}^{d}$, but it can be transferred to the periodic setting in $\mathbb{T}^{d}$ by Poisson summation (cf. [25]).

Let $\psi_{0}$ be a radial bump function such that $\psi_{0}(\xi)=1$ when $[|\xi| \leq 1 / 2] \subset \mathbb{R}^{d}$, and

$$
0 \leq \psi_{0} \leq 1 \text { and } \operatorname{spt} \psi_{0}=[|\xi| \leq 1] \text {. }
$$

Define $\phi_{0}(\xi):=\psi_{0}(\xi / 2)-\psi_{0}(\xi)$. Observe that

$$
0 \leq \varphi_{0} \leq 1 \text { and } \operatorname{spt} \phi_{0}=[1 / 4 \leq\|\xi\| \leq 1]
$$

Now for each $j \in \mathbb{Z}$, define $\psi_{j}:=\left(\psi_{0}\right)_{2^{-j}}$ and $\varphi_{j}:=\left(\varphi_{0}\right)_{2^{-j}}$, where we use the notation

$$
g_{\lambda}(x):=g(\lambda x) \text {. }
$$

for any $\lambda \geq 0$.

In view of the above definitions, clearly $\varphi_{0}:=\psi_{1}-\psi_{0}$ and $\psi_{j+1}=\psi_{j}+\varphi_{j}$, so that

$$
\operatorname{spt} \varphi_{j}=\left[2^{j-2} \leq|\xi| \leq 2^{j}\right]
$$

Moreover, we have

$$
\sum_{j \in \mathbb{Z}} \varphi_{j}(\xi)=1, \text { for } \xi \in \mathbb{R}^{d} \backslash\{\mathbf{0}\}
$$

One can then define

$$
\triangle_{j} g:=\check{\varphi}_{j} * g, \quad \widetilde{\triangle}_{j} g:=\sum_{|j-\ell| \leq 2} \triangle_{\ell} g, \quad S_{m} g:=\sum_{\ell \leq m-3} \triangle_{\ell} g .
$$

We refer to the operators $\triangle_{j}$ as Littlewood-Paley blocks while the operators $S_{m}$ are called the smoothed modal projections.

For functions which are spectrally supported in a compact set, one has the Bernstein inequalities (cf. [26]). We state it here in terms of Littlewood-Paley blocks. 
Lemma 2.3.1 (Bernstein inequalities). Let $1 \leq p \leq q \leq \infty$ and $g \in \mathcal{S}^{\prime}\left(\mathbb{T}^{d}\right)$. Then

$$
C^{-1} 2^{j \alpha}\left\|\triangle_{j} g\right\|_{L^{q}} \leq\left\|(-\Delta)^{\alpha / 2} \triangle_{j} g\right\|_{L^{q}} \leq C 2^{j \alpha+d(1 / p-1 / q)}\left\|\triangle_{j} g\right\|_{L^{p}}
$$

for each $j \in \mathbb{Z}$ and $\alpha \in \mathbb{R}$.

We will also make crucial use of the Littlewood-Paley inequality (cf. [27, 28, 22]).

Lemma 2.3.2 (Littlewood-Paley inequality). Let $1<p<\infty$ and $\alpha \in \mathbb{R}$. Let

$$
\mathscr{S}_{\alpha} g:=\left(\sum_{j \in \mathbb{Z}} 2^{2 \alpha j}\left(\triangle_{j} g\right)^{2}\right)^{1 / 2} .
$$

Suppose $(-\Delta)^{\alpha / 2} g \in L^{p}\left(\mathbb{T}^{2}\right)$. There exists an absolute constant $c_{2.23}=c_{2.23}(p)$ such that

$$
C^{-1}\left\|(-\Delta)^{\alpha / 2} g\right\|_{L^{p}} \leq\left\|\mathscr{S}_{\alpha} g\right\|_{L^{p}} \leq C\left\|(-\Delta)^{\alpha / 2} g\right\|_{L^{p}}
$$

Moreover, $C:=C^{\prime} \max \left\{p,(p-1)^{-1}\right\}$, for some absolute constant $C^{\prime}>0$.

2.4. Modal interpolant observables. For $g$ periodic in $\mathbb{T}^{2}$ with mean zero, if one defines modal projection by

$$
P_{N} g:=\sum_{\substack{\left|k_{1}\right| \leq N \\\left|k_{2}\right| \leq N}} e^{i k \cdot x} \hat{g}(k),
$$

then $P_{N}$ is a bounded operator in $L^{p}$ (cf. [27]) and by Parseval's identity:

$$
\left\|g-P_{N} g\right\|_{L^{2}}^{2} \leq C N^{-2 \alpha}\left\|(-\Delta)^{\alpha / 2} g\right\|_{L^{2}}^{2},
$$

Similarly, by the Bernstein inequalities, the Littlewood-Paley modal interpolant satisifes

$$
\left\|g-S_{m} g\right\|_{L^{2}}^{2} \leq C 2^{-m \alpha}\left\|(-\Delta)^{\alpha / 2} g\right\|_{L^{2}}^{2}
$$

Notice that one can easily make higher-order estimates with the modal interpolant according to $(2.24)$ or $(2.25)$.

Lastly, observe that by definition we have

$$
S_{m} P_{N}=P_{N}, \quad N \leq 2^{m-4} \quad \text { and } \quad P_{N} S_{m}=S_{m}, \quad N \geq 2^{m-3} .
$$

Since both $P_{m}$ and $S_{m}$ are bounded operators in $L^{p}$, we may use $S_{m}$ or $P_{2^{m}}$ as our interpolant.

2.5. Well-posedness and A priori bounds. It is shown in [29, 30, 31] that the problem (2.6) is well-posed and moreover that the equations possess a compact finite-dimensional global attractor, $\mathcal{A}$, when $f$ is time-independent.

Proposition 2.1. Let $u_{0} \in V$ and $f \in L^{\infty}((0, \infty) ; H)$. Then for any $T>0$ the initial value problem (2.6) has a unique solution that satisfies

$$
u \in C([0, T] ; V) \cap L^{2}(0, T ; D(A)) \quad \text { and } \quad \frac{d u}{d t} \in L^{2}(0, T ; H) .
$$


We recall that for any solution $u$ of (2.2) corresponding to $u_{0} \in V$, we have the following bounds satisfied for some $t_{0}=t_{0}\left(u_{0}\right)$ :

$$
\|u(t)\|_{H}^{2} \leq 2 \mathcal{G}^{2}, \quad\|u(t)\|_{V}^{2} \leq 2 \mathcal{G}^{2}, \quad t \geq t_{0},
$$

where $\mathcal{G}$ denotes the Grashof number, which is defined as

$$
\mathcal{G}:=\sup _{t>0}\|f(t)\|_{H}
$$

In particular, given $f \in H$, if $f$ is time-independent, then

$$
\|A u(t)\|_{H}^{2} \leq C(1+\mathcal{G})^{4}, \quad t \geq t_{0}
$$

for some absolute constant $C>0$. The estimates in (2.27) are classical and elementary and can be found in the aforementioned references, while (2.29) can be found in [32].

We will also invoke the following improved estimates for the vorticity (cf. [20]). For this and later, we will use the modified Grashof number as defined by (1.6). In the rescaled variables, they become

$$
\mathcal{G}_{\infty}:=\sup _{t>0}\|f(\cdot, t)\|_{L^{\infty}} \quad \text { and } \quad \widetilde{\mathcal{G}}_{\infty}:=\sup _{t>0}\|F(\cdot, t)\|_{L^{\infty}}
$$

Lemma 2.5.1. Let $f \in L^{\infty}\left((0, \infty) ; L^{\infty}\left(\mathbb{T}^{2}\right)^{2}\right)$ such that $\int_{\mathbb{T}^{2}} f d x=0$. Suppose that $u_{0} \in H$. Then there exists $t_{0}>0$ and an absolute constant $C_{0}>0$ such that for all $p \in[1, \infty)$, we have

$$
\|\nabla \times u(t)\|_{L^{\infty}} \leq C_{0} \mathcal{G}_{\infty} \quad \text { and } \quad\|\nabla u(t)\|_{L^{p}} \leq C_{0} p \mathcal{G}_{\infty}, \quad t \geq t_{0} .
$$

In particular, if $f$ is time-independent and $u_{0} \in \mathcal{A}$, then

$$
\left\|\nabla \times u_{0}\right\|_{L^{\infty}} \leq C_{0} \mathcal{G}_{\infty} .
$$

Finally, let us also recall the following result of Azouani, Olson, and Titi [3] for the data assimilation system (1.2).

Proposition 2.2. Suppose that $v_{0} \in V$ and $f \in L^{\infty}((0, \infty), H)$. There exists an absolute constant $c_{0}>0$ such that if $\mu, m$ satisfy $2^{2 m} \mu^{-1} \geq c_{0}$, then for all $T>0$, the initial value problem (2.3) has a unique solution that satisfies

$$
v \in C([0, T] ; V) \cap L^{2}(0, T ; D(A)) \quad \text { and } \quad \frac{d v}{d t} \in L^{2}(0, T ; H) .
$$

Furthermore, this solution depends continuously on the initial data $v_{0}$ in the $V$ norm.

Proposition 2.3. Let $f \in L^{\infty}((0, \infty) ; H)$. Let $u, v$ be the unique solutions of (2.2), (2.3) corresponding to $u_{0}, v_{0} \in V$, respectively. There exist absolute constants $c_{0}, c_{1}>0$ such that if

$$
\frac{2^{2 m}}{\mu} \geq c_{0} \quad \text { and } \quad \mu \geq c_{1} \mathcal{G}(1+\log (1+\mathcal{G}))
$$

then $\|v(t)-u(t)\|_{V} \rightarrow 0$ exponentially as $t \rightarrow \infty$.

Remark 2.5.1. We will refer to the solutions $u, v$ obtained by Propositions 2.1 and 2.2, respectively, as "strong solutions" of (2.2), (2.3). 


\section{Proof of Theorem 1}

3.1. A priori bounds in $L^{p}$. We demonstrate that one can achieve asymptotic synchronization in $L^{\infty}$ without increasing number of modes with respect to the order of the Grashof number. To do so, we exploit the transport structure of the equation and to accommodate estimates in $L^{p}$, we make use of the Littlewood-Paley modal projection. We will first establish the following estimate for the difference $\nabla \times v-\nabla \times u$.

Proposition 3.1. Let $p \in[2, \infty)$ be an even integer, $\int_{\mathbb{T}^{2}} f d x=0, f \in L^{\infty}\left((0, \infty) ; L^{\infty}\left(\mathbb{T}^{2}\right)^{2}\right)$, and $\nabla \times f \in L^{\infty}\left((0, \infty) ; L^{p}\left(\mathbb{T}^{2}\right)\right)$. Let $u, v$ be smooth solutions of (2.2), (2.3), respectively, and define

$$
\mathfrak{F}_{\infty}\left(t_{0}\right):=\sup _{t>t_{0}}\|\omega(t)\|_{L^{\infty}} \quad \text { with } \quad \omega(t)=\nabla \times u(t) .
$$

There exist $t_{0}>0$, and absolute constants $c_{0}, C_{0}=C_{0}(p), C_{1}$ such that if

$$
\left\|\nabla \times u\left(t_{0}\right)\right\|_{L^{\infty}}+\left\|\nabla \times v\left(t_{0}\right)\right\|_{L^{p}} \leq M, \quad M \geq C_{1} \mathcal{G}_{\infty},
$$

and $\mu, m$ satisfy

$$
\frac{2^{2 m}}{\mu} \geq c_{0} p^{7}
$$

then

$$
\|\nabla \times v(t)-\nabla \times u(t)\|_{L^{p}}^{p} \leq M^{p} e^{-\mu\left(t-t_{0}\right)}+C_{0}(p) \frac{\mathfrak{F}_{\infty}\left(t_{0}\right)^{p}}{\mu^{p / 2}} \int_{t_{0}}^{t} e^{-\mu(t-s)}\|v(s)-u(s)\|_{L^{p}}^{p} d s, \quad t>0 .
$$

Proof. Let $t_{0}$ be given as in Lemma 2.5.1 and $C_{1}>0$ an absolute constant such that $\| \nabla \times$ $u(t) \|_{L^{\infty}} \leq C_{1} \mathcal{G}_{\infty}$ holds for all $t \geq t_{0}$. Then $\mathfrak{F}_{\infty}\left(t_{0}\right)<\infty$ by Lemma 2.5.1. Upon taking differences $\boldsymbol{\delta}=\eta-\omega$ and $\mathbf{w}=v-u$, we have

$$
\partial_{t} \boldsymbol{\delta}-\Delta \boldsymbol{\delta}+\mathbf{w} \cdot \nabla \boldsymbol{\delta}+\mathbf{w} \cdot \nabla \omega+u \cdot \nabla \boldsymbol{\delta}+\mu \boldsymbol{\delta}=\mu\left(\boldsymbol{\delta}-S_{m} \boldsymbol{\delta}\right) .
$$

Suppose $p \geq 2$ is even. We multiply (3.4) by $\delta^{p-1}$ and integrate over $\mathbb{T}^{2}$. Then (2.13) implies

$$
\frac{1}{p} \frac{d}{d t}\|\boldsymbol{\delta}\|_{L^{p}}^{p} \underbrace{-\int_{\mathbb{T}^{2}}(\Delta \boldsymbol{\delta}) \boldsymbol{\delta}^{p-1} d x}_{I}+\mu\|\boldsymbol{\delta}\|_{L^{p}}^{p}=\underbrace{-\int_{\mathbb{T}^{2}}(\mathbf{w} \cdot \nabla \omega) \boldsymbol{\delta}^{p-1} d x}_{I I}+\underbrace{\mu \int_{\mathbb{T}^{2}}\left(\boldsymbol{\delta}-S_{m} \boldsymbol{\delta}\right) \boldsymbol{\delta}^{p-1} d x}_{I I I} .
$$

Observe that $I$ can be rewritten as

$$
I=-\int\left(\partial_{k k} \boldsymbol{\delta}\right) \boldsymbol{\delta}^{p-1} d x=(p-1) \int\left(\partial_{k} \boldsymbol{\delta}\right)^{2} \boldsymbol{\delta}^{p-2} d x=\frac{4}{p p^{\prime}}\left\|\nabla\left(\boldsymbol{\delta}^{p / 2}\right)\right\|_{L^{2}}^{2},
$$

where in the last equality we used the following identity, which is valid for $p$ even:

$$
\frac{2}{p} \nabla\left(\phi^{p / 2}\right)=\phi^{(p-2) / 2} \nabla \phi
$$

For $I I$, we integrate by parts and apply (3.6) again to obtain

$$
I I=(p-1) \int(\mathbf{w} \cdot \nabla \boldsymbol{\delta}) \boldsymbol{\delta}^{p-2} \omega d x=\frac{2}{p^{\prime}} \int\left(\mathbf{w} \cdot \nabla\left(\boldsymbol{\delta}^{p / 2}\right)\right) \boldsymbol{\delta}^{(p-2) / 2} \omega d x .
$$


Then by Cauchy-Schwarz, Hölder's inequality, Young's inequality, and (3.1) we have

$$
\begin{aligned}
|I I| & \leq \frac{2}{p^{\prime}}\|\omega\|_{L^{\infty}}\left(\int|\mathbf{w}|^{2}|\boldsymbol{\delta}|^{p-2} d x\right)^{1 / 2}\left\|\nabla\left(\boldsymbol{\delta}^{p / 2}\right)\right\|_{L^{2}} \\
& \leq \frac{2 p}{p^{\prime}}\|\omega\|_{L^{\infty}}^{2}\|\mathbf{w}\|_{L^{p}}^{2}\|\boldsymbol{\delta}\|_{L^{p}}^{p-2}+\frac{1}{p p^{\prime}}\left\|\nabla\left(\boldsymbol{\delta}^{p / 2}\right)\right\|_{L^{2}}^{2} \\
& \leq \frac{2^{p+1}}{p^{\prime}}\left(\frac{p-2}{\mu}\right)^{p / 2} \mathfrak{F}_{\infty}(0)^{p}\|\mathbf{w}\|_{L^{p}}^{p}+\frac{\mu}{2 p^{\prime}}\|\boldsymbol{\delta}\|_{L^{p}}^{p}+\frac{1}{p p^{\prime}}\left\|\nabla\left(\boldsymbol{\delta}^{p / 2}\right)\right\|_{L^{2}}^{2} .
\end{aligned}
$$

Lastly, using the fact that $\triangle_{j}=\triangle_{j} \widetilde{\triangle}_{j}$ and that $\triangle_{j}, \widetilde{\triangle}_{j}$ are Fourier multipliers, observe that we may rewrite $I I I$ as

$$
\begin{aligned}
I I I & =\mu \sum_{j \geq m-2} \int\left(\triangle_{j} \boldsymbol{\delta}\right) \boldsymbol{\delta}^{p-1} d x \\
& =\mu \sum_{j \geq m-2} \int\left(\triangle_{j} \boldsymbol{\delta}\right) \widetilde{\triangle}_{j}\left(\boldsymbol{\delta}^{p-1}\right) d x \\
& =\mu \sum_{j \geq m-2}\left(\int(-\Delta)^{-1}\left[\left(\triangle_{j} \boldsymbol{\delta}\right)\right](-\Delta)\left[\widetilde{\triangle}_{j}\left(\boldsymbol{\delta}^{p-1}\right)\right] d x\right) \\
& =\mu \sum_{j \geq m-2} 2^{-j}\left(\int 2^{j}\left(\triangle_{j} \nabla(-\Delta)^{-1} \boldsymbol{\delta}\right) \cdot \widetilde{\triangle}_{j} \nabla\left(\boldsymbol{\delta}^{p-1}\right) d x\right),
\end{aligned}
$$

where we have a performed an integration by parts to obtain the last equality. We denote the Riesz transform by $\mathcal{R} \sim \nabla(-\Delta)^{-1 / 2}$. Then by the Cauchy-Schwarz inequality, Hölder's inequality, Lemma 2.3.2, and the Calderón-Zygmund theorem (cf. [33], p. 48) we have

$$
\begin{aligned}
|I I I| & \leq 4 \frac{\mu}{2^{m}} \int\left(\mathscr{S}_{1}(-\Delta)^{-1 / 2} \mathcal{R} \boldsymbol{\delta}\right) \cdot\left(\mathscr{S}_{0} \nabla \boldsymbol{\delta}^{p-1}\right) d x \\
& \leq C p^{2} \frac{\mu}{2^{m}}\|\mathcal{R} \boldsymbol{\delta}\|_{L^{p}}\left\|\nabla\left(\boldsymbol{\delta}^{p-1}\right)\right\|_{L^{p^{\prime}}} \\
& \leq C p^{3} \frac{\mu}{2^{m}}\|\boldsymbol{\delta}\|_{L^{p}}\left\|\nabla\left(\boldsymbol{\delta}^{p-1}\right)\right\|_{L^{p^{\prime}}}
\end{aligned}
$$

Using the fact that $\nabla\left(\boldsymbol{\delta}^{p-1}\right)=(p-1) \boldsymbol{\delta}^{p-2} \nabla \boldsymbol{\delta}$ and applying (3.6), observe that

$$
\left\|\nabla\left(\boldsymbol{\delta}^{p-1}\right)\right\|_{L^{p^{\prime}}} \leq(p-1)\left\|\boldsymbol{\delta}^{(p-2) / 2}\right\|_{L^{q}}\left\|\boldsymbol{\delta}^{(p-2) / 2}|\nabla \boldsymbol{\delta}|\right\|_{L^{2}}=\frac{2}{p^{\prime}}\|\boldsymbol{\delta}\|_{L^{p}}^{(p-2) / 2}\left\|\nabla\left(\boldsymbol{\delta}^{p / 2}\right)\right\|_{L^{2}},
$$

where $1 / 2+1 / q=1 / p^{\prime}$, so that $1 / p+1 / q=1 / 2$ and $q=2 p /(p-2)$. This implies that

$$
|I I I| \leq C \frac{p^{3}}{p^{\prime}} \frac{\mu}{2^{m}}\|\boldsymbol{\delta}\|_{L^{p}}^{p / 2}\left\|\nabla\left(\boldsymbol{\delta}^{p / 2}\right)\right\|_{L^{2}} \leq \frac{\mu}{2 p^{\prime}}\|\boldsymbol{\delta}\|_{L^{p}}^{p}+C^{2} \frac{p^{6}}{p^{\prime}} \frac{\mu}{2^{2 m}}\left\|\nabla\left(\boldsymbol{\delta}^{p / 2}\right)\right\|_{L^{2}}^{2} .
$$

Therefore, returning to (3.5) and applying (3.7), (3.8), and then applying (3.2), (3.3), and (3.1) we obtain

$$
\frac{d}{d t}\|\boldsymbol{\delta}\|_{L^{p}}^{p}+\frac{2}{p^{\prime}}\left\|\nabla\left(\boldsymbol{\delta}^{p / 2}\right)\right\|_{L^{2}}^{2}+\mu\|\boldsymbol{\delta}\|_{L^{p}}^{p} \leq C(p) \frac{\mathfrak{F}_{\infty}(0)^{p}}{\mu^{p / 2}}\|\mathbf{w}\|^{p}
$$


where

$$
C(p):=\left(\frac{p-1}{p-2}\right)^{1 / p}(p-2)^{1 / 2}
$$

An application of Gronwall's inequality and the assumption on $\nabla \times u_{0}, \nabla \times v_{0}$ yields

$$
\|\boldsymbol{\delta}(t)\|_{L^{p}}^{p} \leq M e^{-\mu t}+C(p) \frac{\mathfrak{F}_{\infty}(0)^{p}}{\mu^{p / 2}} \int_{t_{0}}^{t} e^{-\mu(t-s)}\|\mathbf{w}(s)\|_{L^{p}}^{p} d s,
$$

which completes the proof.

3.2. Proof of Theorem 1. We will require the following analog of Lemma 2.5.1.

Lemma 3.2.1. Let $p \in[2, \infty)$ be an even integer and $\nabla \times f \in L^{\infty}\left((0, \infty) ; L^{p}\left(\mathbb{T}^{2}\right)\right)$. Let $u$, $v$ be a smooth solution of (2.2), (2.3), respectively. Suppose that $\nabla \times v_{0} \in L^{p}\left(\mathbb{T}^{2}\right)$. There exists an absolute constant $c_{0}$ such that if $\mu, m$ satisfy

$$
\frac{2^{2 m}}{\mu} \geq c_{0} p^{7}
$$

then

$$
\|\nabla \times v(t)\|_{L^{p}} \leq\left\|\nabla \times v_{0}\right\|_{L^{p}} e^{-(\mu / p) t}+\left(1-e^{-(\mu / p) t}\right) \frac{\widetilde{\mathcal{G}}_{\infty}}{\mu}, \quad t>0 .
$$

In particular, there exists an absolute constant $C_{0}>0$ and $t_{0}>0$ such that

$$
\|\nabla \times v(t)\|_{L^{p}} \leq C_{0} \frac{\widetilde{\mathcal{G}}_{\infty}}{\mu}, \quad t \geq t_{0} .
$$

Proof of Lemma 3.2.1. We proceed as in the proof of Proposition 3.1. Indeed, observe that we may rewrite $(2.5)$ as

$$
\partial_{t} \eta-\Delta \eta+v \cdot \nabla \eta+\mu \eta=F+\mu\left(\eta-S_{m} \eta\right) .
$$

Suppose $p \geq 2$ is even. We multiply (3.11) by $\eta^{p-1}$ and integrate over $\mathbb{T}^{2}$. Then (2.13) implies

$$
\begin{aligned}
\frac{1}{p} \frac{d}{d t}\|\eta\|_{L^{p}}^{p} & -\underbrace{\int_{\mathbb{T}^{2}}(\Delta \eta) \eta^{p-1} d x}_{I}+\mu\|\eta\|_{L^{p}}^{p} \\
& =\underbrace{\int_{\mathbb{T}^{2}} F \eta^{p-1} d x}_{I V}-\underbrace{\int_{\mathbb{T}^{2}}(v \cdot \nabla \eta) \eta^{p-1} d x}_{I I}+\underbrace{\mu \int_{\mathbb{T}^{2}}\left(\eta-S_{m} \eta\right) \eta^{p-1} d x}_{I I I} .
\end{aligned}
$$

Observe that (2.13) implies $I I=0$. We treat $I, I I I$ as in Proposition 3.1, so that we have

$$
\begin{aligned}
I & =\frac{4}{p p^{\prime}}\left\|\nabla\left(\eta^{p / 2}\right)\right\|_{L^{2}}^{2} \\
|I I I| & \leq \frac{\mu}{4 p^{\prime}}\|\eta\|_{L^{p}}^{p}+C \frac{p^{6}}{p^{\prime}} \frac{\mu}{2^{2 m}}\left\|\nabla\left(\eta^{p / 2}\right)\right\|_{L^{2}}^{2} .
\end{aligned}
$$

We are left with $I V$, on which we simply apply Hölder's inequality and Young's inequality.

$$
|I V| \leq\|F(t)\|_{L^{p}}\|\eta\|_{L^{p}}^{p-1}
$$


Therefore, returning to (3.12) and applying $I-I V$ and (3.10), we arrive at

$$
p\|\eta\|_{L^{p}}^{p-1} \frac{d}{d t}\|\eta\|_{L^{p}}+\mu\|\eta\|_{L^{p}}^{p} \leq \frac{d}{d t}\|\eta\|_{L^{p}}^{p}+\frac{2}{p^{\prime}}\left\|\nabla\left(\eta^{p / 2}\right)\right\|_{L^{2}}^{2}+\mu\|\eta\|_{L^{p}}^{p} \leq p \mu \frac{\|F(t)\|_{L^{p}}}{\mu}\|\eta\|_{L^{p}}^{p-1} .
$$

Dividing by $\|\eta\|_{L^{p}}^{p-1}$ and an application of Gronwall's inequality and (2.30) yields

$$
\|\eta(t)\|_{L^{p}} \leq\left\|\eta_{0}\right\|_{L^{p}} e^{-(\mu / p) t}+\frac{\widetilde{\mathcal{G}}_{\infty}}{\mu}\left(1-e^{-(\mu / p) t}\right),
$$

as desired.

Proof of Theorem 1. Observe that $f \in L^{\infty}\left((0, \infty), L^{2}\left(\mathbb{T}^{2}\right)^{2}\right)$ in addition to $\int_{\mathbb{T}^{2}} f d x=0$ imply $f \in L^{\infty}((0, \infty), H)$. Hence, since $u_{0}, v_{0} \in V$, by (1.4) and Propositions 2.1, 2.2, there exist unique global strong solutions $u, v$ to (1.1), (1.2), respectively.

On the other hand, since $\int_{\mathbb{T}^{2}} f d x=0$ and $f \in L^{\infty}\left((0, \infty) ; L^{\infty}\left(\mathbb{T}^{2}\right)^{2}\right)$, by Lemma 2.5.1, there exists $t_{1}=t_{1}\left(u_{0}\right)$ such that

$$
\|\nabla \times u(t)\|_{L^{\infty}} \leq C \mathcal{G}_{\infty}, \quad t \geq t_{1} .
$$

In particular, this implies $\mathfrak{F}_{\infty}(t)<\infty$, for all $t \geq t_{1}$, where $\mathfrak{F}_{\infty}(t)$ is defined in (3.1). Similarly, by (1.4) and Lemma 3.2.1 there exists $t_{2}=t_{2}\left(v_{0}, p\right)$ such that

$$
\|\nabla \times v(t)\|_{L^{p}} \leq C \frac{\widetilde{\mathcal{G}}_{\infty}}{\mu}, \quad t \geq t_{2} .
$$

Let $t_{0}:=\max \left\{t_{1}, t_{2}\right\}$. Then there exists an absolute constant $M>0$, i.e., $M=C\left(\mathcal{G}_{\infty}+\mu^{-1} \widetilde{\mathcal{G}}_{\infty}\right)$, such that

$$
\left\|\nabla \times u\left(t_{0}\right)\right\|_{L^{p}}+\left\|\nabla \times v\left(t_{0}\right)\right\|_{L^{p}} \leq M .
$$

Therefore, by Proposition 3.1 and the Sobolev inequality, it follows that for all $t \geq t_{0}$, we have

$$
\|\nabla \times v(t)-\nabla \times u(t)\|_{L^{p}}^{p} \leq M^{p} e^{-\mu\left(t-t_{0}\right)}+C(p) \frac{\mathfrak{F}_{\infty}\left(t_{0}\right)^{p}}{\mu^{p / 2}} \int_{t_{0}}^{t} e^{-\mu(t-s)}\|v(s)-u(s)\|_{L^{p}}^{p} d s .
$$

Finally, by Proposition 2.3, there exists an absolute constant $c>0$ such that $\|v(s)-u(s)\|_{V} \leq$ $O\left(e^{-c s}\right)$, which implies $\|\nabla \times v(t)-\nabla \times u(t)\|_{L^{p}} \rightarrow 0$ exponentially, as desired.

\section{Analyticity Radius}

To obtain good convergence rates in the Gevrey norm for the solution of the data assimilation system to synchronize with the reference solution, we will require good estimates for the radius of analyticity for solutions to the data assimilation system. We do so here by adapting the technique of Kukavica [20] (see also [34]) to the data assimilation system. We translate our estimate on the radius of analyticity for the data assimilation system into the Gevrey norm setting in Section 5, under which we prove Theorem 2 and establish synchronization. 
Throughout, we will assume that $f$ is given as the restriction of an analytic function $f(x, y, t)+$ $i g(x, y, t), x, y \in \mathbb{T}^{2}$, such that

$$
\begin{aligned}
& M_{f}:=\left(\sup _{t \in[0, \infty),|y| \leq \delta_{f}}\|f(\cdot, y, t)\|_{L^{\infty}}+\|g(\cdot, y, t)\|_{L^{\infty}}\right)<\infty, \\
& M_{F}:=\left(\sup _{t \in[0, \infty),|y| \leq \delta_{f}}\|F(\cdot, y, t)\|_{L^{\infty}}+\|G(\cdot, y, t)\|_{L^{\infty}}\right)<\infty,
\end{aligned}
$$

where

$$
F:=\nabla \times f \quad \text { and } \quad G:=\nabla \times g .
$$

Note that $f+i g$ and $F+i G$ are continuous in $t$ and analytic in $x+i y$. The main result we will prove in this section is the following.

Proposition 4.1. Let $f$ satisfy (4.1) and $\int_{\mathbb{T}^{2}} f d x=0$. Let $u_{0} \in V$ and $\nabla \times v_{0} \in L^{\infty}\left(\mathbb{T}^{2}\right)$ such that $\int_{\mathbb{T}^{2}} v_{0} d x=0$. There exist absolute constants $c_{0}, c_{1}$ such that for $\mu, m$ satisfying

$$
\mu \geq c_{0} \max \left\{\frac{M_{f}^{2 / 3} \widetilde{\mathcal{G}}_{\infty}^{2 / 3}}{\mathcal{G}_{\infty}^{2}}\left(1+\log _{+} \widetilde{\mathcal{G}}_{\infty}\right)^{1 / 3}, \frac{\widetilde{\mathcal{G}}_{\infty}^{2 / 3}}{\mathcal{G}_{\infty}^{2 / 3}}\left(1+\log _{+} \widetilde{\mathcal{G}}_{\infty}\right)^{1 / 3},\left(1+\log _{+} \widetilde{\mathcal{G}}_{\infty}\right)^{3}, \frac{M_{F}}{\mathcal{G}_{\infty}}, \frac{\widetilde{\mathcal{G}}_{\infty}}{\mathcal{G}_{\infty}}\right\}
$$

and

$$
2^{m} \geq c_{1} \mu\left(1+\log _{+} \widetilde{\mathcal{G}}_{\infty}\right)^{2},
$$

there exists $t_{0}>0$ and an absolute constant $c_{2}>0$ such that the unique solution, $\eta(\cdot, t)$, of (2.3) is real-analytic for $t \geq t_{0}$ with uniform radius of analyticity bounded below by

$$
\delta_{*}:=\min \left\{\frac{\widetilde{\mathcal{G}}_{\infty}}{C \mathcal{G}_{\infty} \mu^{3 / 2}}, \delta_{0}, \delta_{f}\right\}, \quad t \geq t_{0},
$$

where $\delta_{0}$ is given in Corollary 4.1.1.1.

Since $\nabla \times v(t)$ is bounded above by $C \mu^{-1} \widetilde{\mathcal{G}}_{\infty}$ for all $t \geq t_{0}$ (see Lemma 3.2.1 and the proof of Proposition 4.1), by interior elliptic estimates (cf. [20]), we have the following higher-order estimates.

Corollary 4.1.1. Under the hypotheses of Proposition 4.1, there exists $t_{0}>0$ and an absolute constant $C_{2}>0$ such that

$$
\left\|\partial^{\beta} \eta(\cdot, t)\right\|_{L^{\infty}\left(\mathbb{T}^{2}\right)} \leq C_{2}|\beta| !\left(\frac{C_{2}}{\delta_{*}}\right)^{|\beta|} \frac{\widetilde{\mathcal{G}}_{\infty}}{\mu}, \quad t \geq t_{0}, \quad \beta \in \mathbb{N}_{0}^{2} .
$$

To prove Proposition 4.1, we consider successive approximations to the analytic extension of (2.5) by linear, inhomogeneous heat equations, whose solutions are analytic in $\mathbb{T}^{2}$. We then establish bounds through a suitable functional, which are uniform with respect to this family, $\mathscr{F}$, of approximations. These uniform bounds will ensure that $\mathscr{F}$ is a normal family in $L^{p}\left(\mathbb{T}^{2}\right)$, and hence, upon passing to a convergent subsequence, that the corresponding limiting function is also analytic over $\mathbb{T}^{2}$ with uniform radius of analyticity bounded below by the quantity asserted 
in Proposition 4.1. We define the approximating solutions and the desired functional in Section 4.1, then establish the requisite bounds for this functional in Section 4.2 (see Lemma 4.2.1).

4.1. Approximate solutions. Let us recall the following fact regarding analyticity of the solution $\omega$ to (2.4). Note that it follows from Lemmas 3.2 and 3.6 in [20].

Lemma 4.1.1. Let $\left\|\omega_{0}\right\|_{L^{\infty}} \leq M_{\infty}$. There exists an absolute constant $K_{0}, K_{1}>0$ such that for

$$
t_{0}:=\frac{M_{\infty}^{4}}{K_{0} M_{f}^{2}\left(1+\log _{+} M_{\infty}\right)} .
$$

Then there exists a unique solution of (2.2) on the interval $\left[0, t_{0}\right]$ corresponding to initial vorticity $\omega_{0}$ such that for every $t \in\left(0, t_{0}\right]$, the uniform space analyticity radius of $\omega(\cdot, t)$ is greater than

$$
\delta_{0}(t)=\min \left\{\frac{t^{1 / 2}}{K_{1}\left(1+M_{\infty} t\left(1+\log _{+} M_{\infty}\right)^{1 / 2}\right)}, \delta_{f}\right\},
$$

and for each $p \in[3 / 2, \infty)$, we have the estimate

$$
\int_{0}^{1} \int_{\mathbb{T}^{2}}\left(\omega(x, s y, t)^{2}+\theta(x, s y, t)^{2}\right)^{p} d x d s \leq C^{2 p} M_{\infty}^{2 p}
$$

for some absolute constant $C>0$, independent of $p$, which holds for all $|y| \leq \delta_{0}(t)$ and $t \in\left(0, t_{0}\right]$, where $\omega+i \theta$ is the analytic extension of $\omega$.

As with Corollary 4.1.1, we have the following higher-order estimates for $\omega$.

Corollary 4.1.1.1. Let $f$ satisfy $M_{f}<\infty$ and $\int_{\mathbb{T}^{2}} f d x=0$. Suppose $u_{0} \in H$. Then there exists $t_{0}>0$ and an absolute constant $K_{2}>0$ such that

$$
\left\|\partial^{\beta} \omega(\cdot, t)\right\|_{L^{\infty}\left(\mathbb{T}^{2}\right)} \leq K_{2}|\beta| !\left(\frac{K_{2}}{\delta_{0}}\right)^{|\beta|} \mathcal{G}_{\infty}, \quad \beta \in \mathbb{N}_{0}^{2}, \quad t \geq t_{0},
$$

where

$$
\delta_{0}:=\min \left\{\delta_{0}\left(t_{*}\right), \delta_{f}\right\}, \quad t_{*}:=\frac{1}{K_{0}\left(1+\log _{+} M_{\infty}\right)^{1 / 2}} \min \left\{\frac{M_{\infty}^{4}}{M_{f}^{2}\left(1+\log _{+} M_{\infty}\right)^{1 / 2}}, \frac{1}{M_{\infty}}\right\} .
$$

Proof of Corollary 4.1.1.1. We apply Lemma 2.5.1 to deduce $\|\omega(t)\|_{L^{\infty}} \leq C \mathcal{G}_{\infty}$ for all $t$ beyond a sufficiently large transient time. Then we apply Lemma 4.1 .1 with $t_{*}$ replacing $t_{0}$ to obtain a uniform lower bound on the analyticity radius of $\omega$ over a fixed interval past the transient time. The uniform bound on $\|\omega(t)\|_{L^{\infty}}$ coupled with a time-translation argument then ensures that this uniform lower bound propagates for all time beyond the transient; we refer to the proof of Proposition 4.1 for additional relevant details.

Remark 4.1.1. Note that in the case where $f$ is time-independent and satisfies $P_{N} f=f$, then one has

$$
M_{F} \sim_{N} M_{f} \sim_{N} \widetilde{\mathcal{G}}_{\infty} \sim_{N} \mathcal{G}_{\infty} \sim_{N} \mathcal{G}
$$


where $A \sim B$ denotes the two-sided relation, $A \leq c B$ and $B \leq c^{\prime} A$ for some constants $c, c^{\prime}$. In particular, by Lemma 2.5.1, after a transient time, $t_{0}$, we may let $M_{\infty} \sim \mathcal{G}$, so that for large $\mathcal{G}$, from Corollary 4.1.1.1 we have

$$
\delta_{0} \geq \frac{1}{K_{1}^{\prime} \mathcal{G}^{1 / 2}\left(1+\log _{+} \mathcal{G}\right)^{1 / 4}}, \quad t \geq t_{0}
$$

for some absolute constant $K_{1}^{\prime}=K_{1}^{\prime}(N)$, exactly as in Theorem 2.1 of [20].

We will consider the following approximating equation to (2.5): Let $\eta^{(0)} \equiv 0$ and $v^{(0)} \equiv 0$ and for $n \geq 1$

$$
\begin{aligned}
& \partial_{t} \eta^{(n)}-\Delta \eta^{(n)}+v^{(n-1)} \cdot \nabla \eta^{(n)}=\nabla \times f-\mu S_{m}\left(\eta^{(n)}-\omega\right), \\
& v^{(n)}=-\nabla^{\perp}(-\Delta)^{-1} \eta^{(n)}, \quad \eta^{(n)}(x, 0)=\eta_{0}(x)=\nabla \times v_{0},
\end{aligned}
$$

For each $t>0, v^{(n)}$ and $\eta^{(n)}$ admit extensions $v^{(n)}+i V^{(n)}$ and $\eta^{(n)}+i \Theta^{(n)}$, respectively, which are due to the linearity of the above system, analytic with real-analyticity radius at least $\delta_{f}$. Note that for $j=1,2$, we have by the Cauchy-Riemann equations

$$
\left\{\begin{array} { l } 
{ \partial _ { y _ { j } } \eta ^ { ( n ) } = - \partial _ { x _ { j } } \Theta ^ { ( n ) } } \\
{ \partial _ { x _ { j } } \eta ^ { ( n ) } = \partial _ { y _ { j } } \Theta ^ { ( n ) } }
\end{array} \quad \left\{\begin{array}{l}
\partial_{y_{j}} v^{(n)}=-\partial_{x_{j}} V^{(n)} \\
\partial_{x_{j}} v^{(n)}=\partial_{y_{j}} V^{(n)}
\end{array}\right.\right.
$$

so that the extended system of (4.8) is given by

$$
\begin{aligned}
& \partial_{t} \eta^{(n)}-\Delta \eta^{(n)}+v_{j}^{(n-1)} \partial_{j} \eta^{(n)}-V_{j}^{(n-1)} \partial_{j} \Theta^{(n)}=F-\mu S_{m}\left(\eta^{(n)}-\omega\right), \quad \eta^{(n)}(x, y, 0)=\eta_{0}(x, y), \\
& \partial_{t} \Theta^{(n)}-\Delta \Theta^{(n)}+v_{j}^{(n-1)} \partial_{j} \Theta^{(n)}+V_{j}^{(n-1)} \partial_{j} \eta^{(n)}=G-\mu S_{m}\left(\Theta^{(n)}-\theta\right), \quad \Theta^{(n)}(x, y, 0)=0,
\end{aligned}
$$

where $F=\nabla \times f$ and $G=\nabla \times g$. Also, we have

$$
\nabla \times V^{(n)}=\Theta^{(n)}, \quad \nabla \cdot V^{(n)}=0, \quad \int_{\mathbb{T}^{2}} V^{(n)}=0 .
$$

Finally, let $\varepsilon>0$ and $\vec{\alpha} \in \mathbb{R}^{2}$. For each $n \geq 0$, define the functional

$$
\Psi_{n}(t):=\int_{0}^{1} \int\left(\eta^{(n)}(x, \vec{\alpha} t s, t)^{2}+\Theta^{(n)}(x, \vec{\alpha} t s, t)^{2}+\varepsilon\right)^{p} d x d s .
$$

In the next section, we will derive uniform bounds for $\Psi_{n}$ and show that these uniform bounds ensure that $\left\{\eta^{(n)}(\cdot, t)+i \Theta^{(n)}(\cdot, t)\right\}_{n \geq 0}$ is normal family. It will then follow as in [20,35] that, upon possibly passing to a subsequence, $\eta^{(n)}+i \Theta^{(n)} \rightarrow \eta+i \Theta$, satisfying (4.9), which satisfies certain lower bounds on its analyticity radius.

4.2. Analyticity radius. We will prove the following.

Lemma 4.2.1. Let $p \in[3 / 2, \infty)$ and $M_{\infty}, M>0$. Suppose that

$$
\left\|\omega_{0}\right\|_{L^{\infty}} \leq M_{\infty} \quad \text { and } \quad\left\|\eta_{0}\right\|_{L^{2 p}} \leq M \text {. }
$$

There exist absolute constants $c_{0}, c_{1}, c_{2}>0$ such that for

$$
t_{0}=\frac{1}{c_{0}} \min \left\{\frac{M_{\infty}^{4}}{M_{f}^{2}\left(1+\log _{+} M_{\infty}\right)}, \frac{\mu M^{2}}{M_{F}^{2}}, \frac{M^{2}}{M_{\infty}^{2} \mu}, 1\right\},
$$


and $\mu, m$ satisfying

$$
2^{m} \geq c_{1} p^{2} \mu^{(p-1) / p}
$$

then there exists a unique solution $\eta(\cdot, t)$ to (2.5) for $0 \leq t \leq t_{0}$, which is real-analytic with uniform radius of spatial analyticity bounded below by

$$
\delta(t):=\min \left\{\frac{t^{1 / 2}}{c_{2}}, \frac{1}{c_{2} p M} \frac{1}{t^{1 / 2}}, \frac{1}{c_{2} p M} \frac{1}{t^{(2 p-3) /(4 p)}}, \frac{1}{c_{2} p M^{2 p /(2 p+3)}} \frac{1}{t^{(2 p-3) /(4 p+6)}}, \delta_{0}(t), \delta_{f}\right\},
$$

for all $t \in\left(0, t_{0}\right)$ where $\delta_{0}(t)$ is given in Lemma 4.1.1. Moreover, $\eta$ is the restriction of an analytic function $\eta(x, y, t)+i \Theta(x, y, t)$ such that

$$
\int_{|y| \leq \delta(t)} \int_{\mathbb{T}^{2}}\left(\eta(x, y, t)^{2}+\Theta(x, y, t)^{2}\right)^{p} d x d y \leq 2^{2 p} \delta(t)^{2} M^{2 p}, \quad t \in\left(0, t_{0}\right] .
$$

To prove Lemma 4.2.1, we will make use of the following Gronwall inequality, which is proved in $[20]$.

Lemma 4.2.2. Let $y_{n}(t) \in C^{1}([0, T])$ be a sequence of non-negative functions satisfying $y_{0}(t) \leq$ $M$ for $0 \leq t \leq T$, and $y_{n}(0) \leq M$ for $n \geq 1$. Suppose that

$$
\frac{d}{d t} y_{n}(t) \leq \sum_{j=1}^{N} K_{j} t^{\alpha_{j}} y_{n}(t)^{\beta_{j}} y_{n-1}(t)^{\gamma_{j}}, \quad t \in[0, T]
$$

where $K_{j}>0, \alpha_{j}>-1, \beta_{j} \in \mathbb{R}, \gamma_{j} \geq 0$, and $\delta>0$ are given constants. Then $y_{n}(t) \leq 2 M$ for all $n=0,1,2, \ldots$ provided that

$$
0 \leq t \leq \min \left\{T, \min _{j=1, \ldots, N}\left(\frac{\alpha_{j}+1}{N K_{j} 2^{\beta_{j}^{+}+\gamma_{j}} M^{\beta_{j}+\gamma_{j}-1}}\right)^{1 /\left(\alpha_{j}+1\right)}\right\},
$$

where $x^{+}=\max \{x, 0\}$.

Let us proceed to the proof of Lemma 4.2.1.

Proof of Lemma 4.2.1. Let $\varepsilon>0, \vec{\alpha} \in \mathbb{R}^{d}$, and $\Psi_{n}(t)$ be given by (4.10). Define

$$
Q_{n, \alpha}^{(\varepsilon)}(x, s, t):=\eta^{(n)}(x, \vec{\alpha} t s, t)^{2}+\Theta^{(n)}(x, \vec{\alpha} t s, t)^{2}+\varepsilon .
$$

For convenience, we will suppress the subscript $\alpha$ and superscript $\varepsilon$. Let $D=\mathbb{T}^{2} \times[0,1]$. Observe then that $\Psi_{n}$ can be rewritten as

$$
\Psi_{n}(t)=\int_{0}^{1} \int Q_{n}(x, s, t)^{p} d x d s=\left\|Q_{n}\right\|_{L^{p}(D)}^{p} .
$$

It follows from (4.9) that

$$
\frac{1}{2 p} \frac{d}{d t} \Psi_{n}(t)+I_{0}=\sum_{k=1}^{6} I_{k}
$$


where

$$
\begin{aligned}
I_{0}= & \int_{0}^{1} \int Q_{n}^{p-1}\left(\left|\nabla \eta^{(n)}\right|^{2}+\left|\nabla \Theta^{(n)}\right|^{2}+\mu\left|\eta^{(n)}\right|^{2}+\mu\left|\Theta^{(n)}\right|^{2}\right) d x d s \\
& +2(p-1) \int_{0}^{1} \int Q_{n}^{p-2}\left|\eta^{(n)} \nabla \eta^{(n)}+\Theta^{(n)} \nabla \Theta^{n}\right|^{2} d x d s .
\end{aligned}
$$

and

$$
\begin{aligned}
& I_{1}=\int_{0}^{1} \int Q_{n}^{p-1}\left(-\eta^{(n)} \nabla \Theta^{(n)}+\Theta^{(n)} \nabla \eta^{(n)}\right) \cdot \vec{\alpha} s d x d s, \\
& I_{2}=\int_{0}^{1} \int Q_{n}^{p-1}\left(\eta^{(n)} \nabla \eta^{(n)}+\Theta^{(n)} \nabla \Theta^{(n)}\right) \cdot v^{(n-1)} d x d s, \\
& I_{3}=\int_{0}^{1} \int Q_{n}^{p-1}\left(\eta^{(n)} \nabla \Theta^{(n)}-\Theta^{(n)} \nabla \eta^{(n)}\right) \cdot V^{(n-1)} d x d s, \\
& I_{4}=\int_{0}^{1} \int Q_{n}^{p-1}\left(\eta^{(n)} F+\Theta^{(n)} G\right) d x d s, \\
& I_{5}=\mu \sum_{j \leq m-3} \int_{0}^{1} \int Q_{n}^{p-1}\left(\eta^{(n)} \triangle_{j} \omega+\Theta^{(n)} \triangle_{j} \theta\right) d x d s, \\
& I_{6}=\mu \sum_{j \geq m-2} \int_{0}^{1} \int Q_{n}^{p-1}\left(\eta^{(n)} \triangle_{j} \eta^{(n)}+\Theta^{(n)} \triangle_{j} \Theta^{(n)}\right) d x d s .
\end{aligned}
$$

It suffices to consider $I_{5}$ and $I_{6}$. Indeed, observe that $I_{2}=0$, while the terms $I_{1}-I_{4}$ will be estimated exactly as in [35]. Collecting these estimates, we arrive at

$$
\begin{aligned}
I_{1} \leq & \frac{1}{12} I_{0}+C|\vec{\alpha}|^{2} \Psi_{n}(t) \\
I_{3} \leq & \frac{1}{12} I_{0}+C p^{2}|\vec{\alpha}|^{2} t^{2} \Psi_{n-1}(t)^{1 / p} \Psi_{n}(t) \\
& +C p^{2}|\vec{\alpha}|^{4 p /(2 p-3)} t^{4 p /(2 p-3)}\left(1+|\vec{\alpha}|^{2} t^{2}\right)^{3 /(2 p-3)} \Psi_{n-1}(t)^{2 /(2 p-3)} \Psi_{n}(t), \\
I_{4} \leq & \frac{1}{12} I_{0}+C \mu^{-1} M_{F}^{2} \Psi_{n}(t)^{(p-1) / p}
\end{aligned}
$$


Let us treat $I_{5}$. Observe that the Cauchy-Schwarz inequality, Young's inequality, Hölder's inequality, and by definition of $\Psi_{n},(4.19)$, and Lemma 4.1 .1 we estimate

$$
\begin{aligned}
\left|I_{5}\right| & \leq \mu \int_{0}^{1}\left|\int Q_{n}^{(p-1) / 2} \eta^{(n)} Q_{n}^{(p-1) / 2} S_{m} \omega+Q_{n}^{(p-1) / 2} \Theta^{(n)} Q_{n}^{(p-1) / 2} S_{m} \theta d x\right| d s \\
& \leq \int_{0}^{1}\left[\int Q_{n}^{p-1}\left(\mu\left|\eta^{(n)}\right|^{2}+\mu\left|\Theta^{(n)}\right|^{2}\right) d x\right]^{1 / 2}\left[\int Q_{n}^{p-1}\left(\mu\left|S_{m} \omega\right|^{2}+\mu\left|S_{m} \theta\right|^{2}\right) d x\right]^{1 / 2} d s \\
& \leq \frac{1}{4} I_{0}+C \mu \int_{0}^{1} \int Q_{n}^{p-1}\left(\left|S_{m} \omega\right|^{2}+\left|S_{m} \theta\right|^{2}\right) d x d s \\
& \leq \frac{1}{4} I_{0}+C \mu \int_{0}^{1}\left(\int Q_{n}^{p} d x\right)^{(p-1) / p}\left(\int\left(\left|S_{m} \omega\right|^{2}+\left|S_{m} \theta\right|^{2}\right)^{p} d x\right)^{1 / p} d s \\
& \leq \frac{1}{4} I_{0}+C \mu \Psi_{n}(t)^{(p-1) / p}\left(\int_{0}^{1} \int\left(|\omega|^{2}+|\theta|^{2}\right)^{p} d x d s\right)^{1 / p} \\
& \leq \frac{1}{4} I_{0}+C \mu \Psi_{n}(t)^{(p-1) / p} M_{\infty}^{2} .
\end{aligned}
$$

To estimate $I_{6}$, let $\phi \in\left\{\eta^{(n)}, \Theta^{(n)}\right\}$. Let us first observe that since $\left(I-S_{m}\right) \phi$ is spectrally supported outside of a shell of radius $\gtrsim 2^{m}$, we have

$$
\left(I-S_{m}\right) \phi=\left(I-S_{m}\right)\left(I-S_{m-2}\right) \phi=\left(I-S_{m}\right) \widetilde{\phi}
$$

where $\widetilde{\phi}=\left(I-S_{m-2}\right) \phi$. We may then make the following calculations:

$$
\begin{aligned}
& \int Q_{n}^{p-1} \widetilde{\phi} \triangle_{j} \widetilde{\phi} d x \\
= & \int(-\Delta)\left(Q_{n}^{p-1} \widetilde{\phi}\right)(-\Delta)^{-1} \triangle_{j} \widetilde{\phi} d x \\
= & -\int \partial_{k} \partial_{k}\left(Q_{n}^{p-1} \widetilde{\phi}\right)(-\Delta)^{-1} \triangle_{j} \widetilde{\phi} d x \\
= & \int \partial_{k}\left(Q_{n}^{p-1} \widetilde{\phi}\right) \mathcal{R}_{k}(-\Delta)^{-1 / 2} \triangle_{j} \widetilde{\phi} d x \\
= & 2(p-1) \int Q_{n}^{p-2}\left(\eta^{(n)} \partial_{k} \eta^{(n)}+\Theta^{(n)} \partial_{k} \Theta^{(n)}\right) \widetilde{\phi} \triangle_{j} \mathcal{R}_{k} \Lambda^{-1} \widetilde{\phi} d x+\int Q_{n}^{p-1} \partial_{k} \widetilde{\phi} \triangle_{j} \mathcal{R}_{k} \Lambda^{-1} \widetilde{\phi} d x \\
= & 2(p-1) \int Q_{n}^{(p-2) / 2}\left(\eta^{(n)} \partial_{k} \eta^{(n)}+\Theta^{(n)} \partial_{k} \Theta^{(n)}\right) Q_{n}^{(p-2) / 2} \widetilde{\phi} \triangle_{j} \mathcal{R}_{k} \Lambda^{-1} \widetilde{\phi} d x \\
& +\int Q_{n}^{(p-1) / 2}\left(\partial_{k} \widetilde{\phi}\right) Q_{n}^{(p-1) / 2} \triangle_{j} \mathcal{R}_{k} \Lambda^{-1} \widetilde{\phi} d x .
\end{aligned}
$$


Using the notation $\Lambda:=(-\Delta)^{1 / 2}$, it follows that

$$
\begin{aligned}
& \left|\int Q_{n}^{p-1} \widetilde{\phi} \triangle_{j} \widetilde{\phi} d x\right| \\
& \leq 2(p-1)\left(\int Q_{n}^{p-2}\left|\eta^{(n)} \nabla \eta^{(n)}+\Theta^{(n)} \nabla \Theta^{(n)}\right|^{2} d x\right)^{1 / 2}\left(\int Q_{n}^{p-2}(\widetilde{\phi})^{2}\left|\triangle_{j} \Lambda^{-1} \mathcal{R} \widetilde{\phi}\right|^{2} d x\right)^{1 / 2} \\
& +\left(\int Q_{n}^{p-1}|\nabla \widetilde{\phi}|^{2} d x\right)^{1 / 2}\left(\int Q_{n}^{p-1}\left|\triangle_{j} \Lambda^{-1} \mathcal{R} \widetilde{\phi}\right|^{2} d x\right)^{1 / 2} .
\end{aligned}
$$

Integrating over $[0,1]$ and applying the Cauchy-Schwarz inequality we have

$$
\begin{aligned}
& \sum_{j \geq m-2}\left|\int_{0}^{1} \int Q_{n}^{p-1} \widetilde{\phi} \triangle_{j} \widetilde{\phi} d x d s\right| \\
& \leq C \frac{2(p-1)}{2^{m}}\left(\int_{0}^{1} \int Q_{n}^{p-2}\left|\eta^{(n)} \nabla \eta^{(n)}+\Theta^{(n)} \nabla \Theta^{(n)}\right|^{2} d x\right)^{1 / 2}\left(\int_{0}^{1} \int Q_{n}^{p-2}\left|\mathscr{S}_{1}\left(\Lambda^{-1} \mathcal{R} \widetilde{\phi}\right)\right|^{2} \widetilde{\phi}^{2} d x d s\right)^{1 / 2} \\
& +C 2^{-m}\left(\int_{0}^{1} \int Q_{n}^{p-1}|\nabla \widetilde{\phi}|^{2} d x\right)^{1 / 2}\left(\int_{0}^{1} \int Q_{n}^{p-1}\left|\mathscr{S}_{1}\left(\Lambda^{-1} \mathcal{R} \widetilde{\phi}\right)\right|^{2} d x d s\right)^{1 / 2}
\end{aligned}
$$

Observe that by Hölder's inequality we have

$$
\begin{aligned}
& \int_{0}^{1} \int Q_{n}^{p-2}\left(\mathscr{S}_{1}\left(\mathcal{R} \Lambda^{-1} \widetilde{\phi}\right)\right)^{2} \widetilde{\phi}^{2} d x d s \leq\left\|Q_{n}\right\|_{L^{p}(D)}^{p-2}\left\|\phi^{2}\right\|_{L^{p}(D)}\left(\int_{0}^{1}\left\|\mathscr{S}_{1} \mathcal{R} \Lambda^{-1} \varphi\right\|_{L^{2 p}\left(\mathbb{T}^{2}\right)}^{2 p} d s\right)^{1 / p} \\
& \int_{0}^{1} \int Q_{n}^{p-1}\left(\mathscr{S}_{1}\left(\Lambda^{-1} \mathcal{R} \widetilde{\phi}\right)\right)^{2} d x d s \leq\left\|Q_{n}\right\|_{L^{p}(D)}^{p-1}\left(\int_{0}^{1}\left\|\mathscr{S}_{1} \Lambda^{-1} \mathcal{R} \widetilde{\phi}\right\|_{L^{2 p}\left(\mathbb{T}^{2}\right)}^{2 p} d s\right)^{1 / p} .
\end{aligned}
$$

By Proposition 2.3.2 and the Calderón-Zygmund theorem we have

$$
\left\|\mathscr{S}_{1} \Lambda^{-1} \mathcal{R} \widetilde{\phi}\right\|_{L^{2 p}\left(\mathbb{T}^{2}\right)}^{2 p} \leq C p^{2 p}\|\mathcal{R} \widetilde{\phi}\|_{L^{2 p}\left(\mathbb{T}^{2}\right)}^{2 p} \leq C p^{4 p}\|\phi\|_{L^{2 p}\left(\mathbb{T}^{2}\right)}^{2 p} .
$$

Combining (4.22) and (4.23) and returning to (4.21) we arrive at

$$
\begin{aligned}
& \sum_{j \geq m-2}\left|\int_{0}^{1} \int Q_{n}^{p-1} \widetilde{\phi} \triangle_{j} \widetilde{\phi} d x d s\right| \\
& \leq \frac{C p^{2}}{2^{m}} 2(p-1)\left(\int_{0}^{1} \int Q_{n}^{p-2}\left|\eta^{(n)} \nabla \eta^{(n)}+\Theta^{(n)} \nabla \Theta^{(n)}\right|^{2} d x\right)^{1 / 2}\left\|Q_{n}\right\|_{L^{p}(D)}^{(p-2) / 2}\left\|\varphi^{2}\right\|_{L^{p}(D)} \\
& +\frac{C p^{2}}{2^{m}}\left(\int_{0}^{1} \int Q_{n}^{p-1}|\nabla \widetilde{\phi}|^{2} d x\right)^{1 / 2}\left\|Q_{n}\right\|_{L^{p}(D)}^{(p-1) / 2}\left\|\phi^{2}\right\|_{L^{p}(D)}^{1 / 2} .
\end{aligned}
$$

By Young's inequality we have

$$
\mu \sum_{j \geq m-2}\left|\int_{0}^{1} \int Q_{n}^{p-1} \widetilde{\phi} \triangle_{j} \widetilde{\phi} d x d s\right| \leq \frac{1}{16} I_{0}+\frac{C p^{4} \mu^{2}}{2^{2 m}}\left(\left\|Q_{n}\right\|_{L^{p}(D)}^{p-2}\left\|\varphi^{2}\right\|_{L^{p}(D)}^{2}+\left\|Q_{n}\right\|_{L^{p}(D)}^{p-1}\left\|\varphi^{2}\right\|_{L^{p}(D)}\right)
$$


Thus, upon summing over $\phi \in\left\{\eta^{(n)}, \Theta^{(n)}\right\}$, applying Young's inequality, then using (4.13), we have

$$
\begin{aligned}
I_{6} \leq & \frac{1}{8} I_{0}+\frac{C p^{4} \mu^{2-2 / p}}{2^{2 m}} \Psi_{n}(t)^{(p-2) / p}\left(\mu \int_{0}^{1} \int\left(\left(\eta^{(n)}\right)^{2}+\left(\Theta^{(n)}\right)^{2}\right)^{p} d x d s\right)^{2 / p} \\
& +\frac{C p^{4} \mu^{2-1 / p}}{2^{2 m}} \Psi_{n}(t)^{(p-1) / p}\left(\mu \int_{0}^{1} \int\left(\left(\eta^{(n)}\right)^{2}+\left(\Theta^{(n)}\right)^{2}\right)^{p} d x d s\right)^{1 / p} \\
\leq & \frac{1}{8} I_{0}+\Psi_{n}(t)+C \frac{p^{2 p-1} \mu^{p-1}}{2^{m p}} I_{0}+C \frac{p^{4 p-1} \mu^{2 p-2}}{2^{2 m p}} I_{0} \\
\leq & \frac{1}{4} I_{0}+\Psi_{n}(t) .
\end{aligned}
$$

Note that to arrive at the second inequality, we used the fact that

$$
\mu \int_{0}^{1} \int\left(\left(\eta^{(n)}\right)^{2}+\left(\Theta^{(n)}\right)^{2}\right)^{p} d x d s \leq \int_{0}^{1} \int Q_{n}^{p-1}\left(\mu\left(\eta^{(n)}\right)^{2}+\mu\left(\Theta^{(n)}\right)^{2}\right) d x d s \leq I_{0} .
$$

By combining the estimates for $I_{1}-I_{6}$, we arrive at

$$
\begin{aligned}
\frac{1}{2 p} \frac{d}{d t} \Psi_{n}(t) \leq & C|\vec{\alpha}|^{2} \Psi_{n}(t)+C p^{2}|\alpha|^{2} t^{2} \Psi_{n-1}(t)^{1 / p} \Psi_{n}(t) \\
& +C p^{2}|\vec{\alpha}|^{4 p /(2 p-3)} t^{4 p /(2 p-3)}\left(1+|\vec{\alpha}|^{2} t^{2}\right)^{3 /(2 p-3)} \Psi_{n-1}(t)^{2 /(2 p-3)} \Psi_{n}(t) \\
& +C \mu^{-1} M_{F}^{2} \Psi_{n}(t)^{(p-1) / p}+C \mu \Psi_{n}(t)^{(p-1) / p} M_{\infty}^{2}+\Psi_{n}(t) .
\end{aligned}
$$

Let $\Phi_{n}(t):=\Psi_{n}(t)^{1 /(2 p)}$. It then follows that

$$
\begin{aligned}
\frac{d}{d t} \Phi_{n}(t) \leq & C|\vec{\alpha}|^{2} \Phi_{n}(t)+C p^{2}|\vec{\alpha}|^{2} t^{2} \Phi_{n-1}(t)^{2} \Phi_{n}(t) \\
& +C p^{2}|\vec{\alpha}|^{4 p /(2 p-3)} t^{4 p /(2 p-3)} \Phi_{n-1}(t)^{4 p /(2 p-3)} \Phi_{n}(t) \\
& +C p^{2}|\vec{\alpha}|^{(4 p+6) /(2 p-3)} t^{(4 p+6) /(2 p-3)} \Phi_{n-1}(t)^{4 p /(2 p-3)} \Phi_{n}(t) \\
& +C \mu^{-1} M_{F}^{2} \Phi_{n}(t)^{-1}+C \mu M_{\infty}^{2} \Phi_{n}(t)^{-1}+C \Phi_{n}(t) .
\end{aligned}
$$

Now observe that

$$
\Phi_{n}(0)=\left(\int\left(\eta_{0}(x)^{2}+\varepsilon\right)^{p}\right)^{1 /(2 p)}
$$

By Lemma 4.2.2, after sending $\varepsilon \rightarrow 0$, we may deduce

$$
\left.\int_{0}^{1} \int_{\mathbb{T}^{2}}\left(\eta^{(n)}(x, \vec{\alpha} s t, t)^{2}+\Theta^{(n)}(x, \vec{\alpha} s, t)^{2}\right)\right)^{p} d x d s \leq 2^{2 p} M^{2 p},
$$

for all $t \geq 0$ such that $|\vec{\alpha}| t \leq \delta_{f}$ and

$$
\begin{aligned}
t \leq \min \{ & \frac{1}{C|\vec{\alpha}|^{2}}, \frac{1}{C p^{2 / 3}|\vec{\alpha}|^{2 / 3} M^{2 / 3}}, \frac{1}{C p^{2 / 3}|\vec{\alpha}|^{4 p /(6 p-3)} M^{4 p /(6 p-3)}}, \\
& \left.\frac{1}{C p^{2 / 3}|\vec{\alpha}|^{(4 p+6) /(6 p+3)} M^{4 p /(6 p+3)}}, \frac{\mu M^{2}}{C M_{F}^{2}}, \frac{M_{\infty}^{4}}{K_{0} M_{f}^{2}\left(1+\log _{+} M_{\infty}\right)}, \frac{M^{2}}{C \mu M_{\infty}^{2}}, \frac{1}{C}\right\} .
\end{aligned}
$$


Let $y=\vec{\alpha} t$. Observe that $|y| \leq \delta_{f}$. Thus, upon choosing $|\vec{\alpha}|$ sufficiently small, it follows from (4.26) that $|y| \leq \delta(t)$ for $t \in\left(0, t_{0}\right]$, where $t_{0}$ is given by (4.12) and $\delta(t)$ is given by (4.14). In particular, we have

$$
|y| \leq \min \left\{\frac{t^{1 / 2}}{C}, \frac{1}{C p M} \frac{1}{t^{1 / 2}}, \frac{1}{C p M} \frac{1}{t^{(2 p-3) /(4 p)}}, \frac{1}{C p M^{2 p /(2 p+3)}} \frac{1}{t^{(2 p-3) /(4 p+6)}}, \delta_{0}(t), \delta_{f}\right\}, \quad t \in\left(0, t_{0}\right] .
$$

Since $|y| \leq \delta(t)$ for $t \in\left(0, t_{0}\right],(4.25)$ can be rewritten as

$$
\left.\int_{0}^{1} \int_{\mathbb{T}^{2}}\left(\eta^{(n)}(x, s y, t)^{2}+\Theta^{(n)}(x, s y, t)^{2}\right)\right)^{p} d x d s \leq 2^{2 p} M^{2 p}
$$

Upon rescaling and using the fact that $|y| \leq \delta(t)$, the following estimate holds

$$
\left.\int_{0}^{\delta(t)} \int_{\mathbb{T}^{2}}\left(\eta^{(n)}\left(x, s \frac{\vec{\alpha}}{|\vec{\alpha}|}, t\right)^{2}+\Theta^{(n)}\left(x, s \frac{\vec{\alpha}}{|\vec{\alpha}|}, t\right)^{2}\right)\right)^{p} d x d s \leq 2^{2 p} \delta(t) M^{2 p} .
$$

It follows from changing coordinates, the Fubini-Tonelli theorem, and (4.27) that

$$
\begin{aligned}
& \left.\int_{|y| \leq \delta(t)} \int_{\mathbb{T}^{2}}\left(\eta^{(n)}(x, y, t)^{2}+\Theta^{(n)}(x, y, t)^{2}\right)\right)^{p} d x d y \\
& \left.=\int_{0}^{\delta(t)} \int_{\partial B(0,1)} \int_{\mathbb{T}^{2}}\left(\eta^{(n)}(x, r y, t)^{2}+\Theta^{(n)}(x, r y, t)^{2}\right)\right)^{p} d x d y r d r \\
& \left.=\int_{\partial B(0,1)} \int_{0}^{\delta(t)} \int_{\mathbb{T}^{2}}\left(\eta^{(n)}(x, r y, t)^{2}+\Theta^{(n)}(x, r y, t)^{2}\right)\right)^{p} d x r d r d y \leq(2 \pi) 2^{2 p} \delta(t)^{2} M^{2 p}
\end{aligned}
$$

Since (4.28) is uniform in $n$, we may deduce that, upon possibly passing to a subsequence, $\left(\eta^{(n)}, \Theta^{(n)}\right)$ converges uniformly over compact subsets of $\mathbb{T}^{2} \times\left[0, t_{0}\right]$ to a solution $(\eta, \Theta)$ of the analytic extension of (2.5), which is analytic with the asserted lower bound on the uniform real-analyticity radius of the lemma (cf. [34] for additional details).

Now we specialize in Lemma 4.2.1.

Lemma 4.2.3. Let $u_{0}, v_{0}$ be periodic over $\mathbb{T}^{2}$ and have zero mean. Let $\mu, M, M_{\infty}>0$. There exists $p_{0}>1$, depending on $M$, such that if $u_{0}, v_{0}$ satisfy

$$
\left\|\nabla \times u_{0}\right\|_{L^{\infty}} \leq M_{\infty} \quad \text { and } \quad\left\|\nabla \times v_{0}\right\|_{L^{p_{0}}} \leq M
$$

then there exist absolute constants $c_{0}, c_{1}, c_{2}, c_{3}>0$ such that if $\mu>0$ satisfies

$$
\mu \geq c_{0} \max \left\{\frac{M_{f}^{2} M^{2}}{M_{\infty}^{6}}\left(1+\log _{+} M\right), \frac{M_{F}}{M_{\infty}}, 1\right\}
$$

and $m$ satisfies $2^{m} \geq c_{1} \mu^{1-1 / p_{0}}\left(1+\log _{+} M\right)^{2}$, then for $t_{0}:=\frac{M^{2}}{c_{3} M_{\infty}^{2} \mu}$, the unique solution $\eta(\cdot, t)$ of (2.5) is real-analytic on $\left(0, t_{0}\right]$ with uniform radius of analyticity bounded below by

$$
\delta(t) \geq \min \left\{\frac{t^{1 / 2}}{c_{2}}, \frac{1}{c_{2}\left(1+\log _{+} M\right) t^{1 / 2}}, \delta_{0}(t), \delta_{f}\right\}, \quad t \in\left(0, t_{0}\right] .
$$

where $\delta_{0}(t)$ is given in Lemma 4.1.1. 
Proof. Let $M>0$ be given and let $p_{0}=C\left(1+\log _{+} M\right)$ for $C>0$ sufficiently large so that

$$
M^{3 /\left(2 p_{0}-3\right)} \leq C
$$

for some $C>0$. Suppose that

$$
\left\|\nabla \times u_{0}\right\|_{L^{\infty}} \leq M_{\infty} \quad \text { and } \quad\left\|\nabla \times v_{0}\right\|_{L^{p_{0}}} \leq M
$$

For $c_{0}$ sufficiently large in (4.29), we have

$$
\mu>\max \left\{C \frac{M_{f}^{2} M^{2}}{M_{\infty}^{6}}\left(1+\log _{+} M\right), C \frac{M_{F}}{M_{\infty}}, C\right\} .
$$

By assumption on $\mu$ and Lemma 4.2.1, for $t_{0}>0$ given by

$$
t_{0}=\frac{M^{2}}{C M_{\infty}^{2} \mu}
$$

the unique solution $\eta(\cdot, t)$ of $(2.5)$ exists for $t \in\left[0, t_{0}\right]$ and is real-analytic with uniform radius of spatial analyticity bounded below by

$$
\delta(t)=\min \left\{\frac{t^{1 / 2}}{C}, \frac{1}{C p_{0} M} \frac{1}{t^{1 / 2}}, \frac{1}{C p_{0} M} \frac{1}{t^{\left(p_{0}-3\right) /\left(2 p_{0}\right)}}, \frac{1}{C p_{0} M^{p_{0} /\left(p_{0}+3\right)}} \frac{1}{t^{\left(p_{0}-3\right) /\left(2 p_{0}+6\right)}}, \delta_{0}(t), \delta_{f}\right\},
$$

for $t \in\left(0, t_{0}\right]$. By Young's inequality we may estimate

$$
\begin{aligned}
& C p_{0} M t^{\left(p_{0}-3\right) /\left(2 p_{0}\right)} \leq C p_{0} M^{2 p_{0} /\left(2 p_{0}-3\right)} t^{1 / 2}+t^{-1 / 2} \leq 2 \max \left\{C p_{0}\left(M^{2 p_{0} /\left(2 p_{0}-3\right)}+M\right) t^{1 / 2}, t^{-1 / 2}\right\} \\
& C p_{0} M t^{\left(p_{0}-3\right) /\left(2 p_{0}+6\right)} \leq C p_{0} M t^{1 / 2}+t^{-1 / 2} \leq 2 \max \left\{C p_{0}\left(M^{2 p_{0} /\left(2 p_{0}-3\right)}+M\right) t^{1 / 2}, t^{-1 / 2}\right\} .
\end{aligned}
$$

Thus, after possibly making $C$ larger, it follows that

$$
\delta(t) \geq \min \left\{\frac{t^{1 / 2}}{C}, \frac{1}{C p_{0} M} \frac{1}{t^{1 / 2}}, \frac{1}{C p_{0} M\left(M^{3 /\left(2 p_{0}-3\right)}+1\right)} \frac{1}{t^{1 / 2}}, \delta_{0}(t), \delta_{f}\right\}, \quad t \in\left(0, t_{0}\right] .
$$

Hence, from (4.31) it follows that

$$
\delta(t) \geq \min \left\{\frac{t^{1 / 2}}{C}, \frac{1}{C\left(1+\log _{+} M\right) t^{1 / 2}}, \delta_{0}(t), \delta_{f}\right\}
$$

for $t \in\left(0, t_{0}\right]$, as desired.

\subsection{Proof of Proposition 4.1.}

Proof of Proposition 4.1. Let $C_{0}, c_{0}, c_{1}$ be larger than $4 \pi^{2}$ multiplied by constants appearing in Lemma 2.5.1 and 3.2.1. Let $c_{2}$ be the minimum of the $K_{1}$ in Lemma 4.1.1, and the respective constants appearing in 3.2.1 and 4.2.3. Suppose

$$
\mu \geq C \max \left\{\frac{M_{f}^{2 / 3} \widetilde{\mathcal{G}}_{\infty}^{2 / 3}}{\mathcal{G}_{\infty}^{2}}\left(1+\log _{+} \widetilde{\mathcal{G}}_{\infty}\right)^{1 / 3}, \frac{M_{F}}{\mathcal{G}_{\infty}}, \frac{\widetilde{\mathcal{G}}_{\infty}}{\mathcal{G}_{\infty}}, \frac{\widetilde{\mathcal{G}}_{\infty}^{2 / 3}}{\mathcal{G}_{\infty}^{2 / 3}}\left(1+\log _{+} \widetilde{\mathcal{G}}_{\infty}\right)^{1 / 3},\left(1+\log _{+} \widetilde{\mathcal{G}}_{\infty}\right)^{3}, 1\right\}
$$

for some $C>0$ to be determined. 
Since $f$ is mean-zero and satisfies (4.1), we have $f \in L^{\infty}((0, \infty) ; H)$ as well as $\nabla \times f \in$ $L^{\infty}\left((0, \infty), L^{p}\left(\mathbb{T}^{2}\right)\right)$, for all $p \in[1, \infty]$. Since $u_{0} \in V \subset H$, by Lemma 2.5.1, there exists $t_{1}>0$ such that

$$
\|\nabla \times u(t)\|_{L^{\infty}} \leq C_{0} \mathcal{G}_{\infty}=: M_{\infty}, \quad t \geq t_{1}
$$

On the other hand, since $\nabla \times v_{0} \in L^{\infty}\left(\mathbb{T}^{2}\right)$ we have $\nabla \times v_{0} \in L^{p}\left(\mathbb{T}^{2}\right)$ for all $p \in[1, \infty]$. In particular, we choose $p_{0}=C\left(1+\log _{+} M\right)$ with $C>0$ so that $p_{0}$ is even. Thus, by Lemma 3.2.1 we have

$$
\|\nabla \times v(t)\|_{L^{p}} \leq C_{0} \frac{\widetilde{\mathcal{G}}_{\infty}}{\mu}=: M, \quad t \geq t_{2} .
$$

Note that (3.10) is implied by (4.3) as long as $\mu \geq C\left(1+\log _{+} \widetilde{\mathcal{G}}_{\infty}\right)^{3}$, which is satisfied by (4.2).

Now let $t_{3}:=\max \left\{t_{1}, t_{2}\right\}$. It follows that

$$
\|\nabla \times u(t)\|_{L^{\infty}} \leq M_{\infty} \quad \text { and } \quad\|\nabla \times v(t)\|_{L^{p}} \leq M, \quad t \geq t_{3}
$$

for all $p \in(1, \infty)$. By Corollary 4.1.1.1, we have that the unique solution $\omega\left(\cdot, \tau+t_{3}\right)$ of $(2.2)$ with $\omega_{0}(\cdot):=\omega\left(\cdot, t_{3}\right)$, is real-analytic for all $\tau>0$ with analyticity radius bounded below by $\delta_{0}$ given by (4.7).

On the other hand, by Lemma 4.2.3, for

$$
\tau_{0}:=M^{2}\left(C M_{\infty}^{2} \mu\right)^{-1}=\frac{\widetilde{\mathcal{G}}_{\infty}^{2}}{C \mu^{3} \mathcal{G}_{\infty}^{2}},
$$

where $C>0$ is some sufficiently large absolute constant, the unique solution $\eta\left(\cdot, t+t_{3}\right)$ of $(2.3)$ with $\eta_{0}(\cdot):=\eta\left(\cdot, t_{3}\right)$, is real-analytic for $t \in\left(0, \tau_{0}\right]$ with uniform radius of analyticity bounded below by

$$
\delta(t)=\min \left\{\frac{t^{1 / 2}}{C}, \frac{1}{C\left(1+\log _{+} M\right) t^{1 / 2}}, \delta_{0}, \delta_{f}\right\}, \quad t \in\left(0, \tau_{0}\right] .
$$

In particular, since we chose $\mu \geq C \widetilde{\mathcal{G}}_{\infty}^{2 / 3} \mathcal{G}_{\infty}^{-2 / 3}\left(1+\log _{+} \widetilde{\mathcal{G}}_{\infty}\right)^{1 / 3}$, we have

$$
\delta\left(\tau_{0}\right) \geq \min \left\{\frac{\widetilde{\mathcal{G}}_{\infty}}{C \mathcal{G}_{\infty} \mu^{3 / 2}}, \delta_{0}, \delta_{f}\right\}
$$

upon making $C$ possibly larger.

Since (4.33) holds for $t \geq t_{3}+\ell \tau_{0}$ for each integer $\ell \geq 0$, we may apply Lemma 4.2 .3 with initial data $\nabla \times u(s), \nabla \times v(s)$ for each $s \in\left(t_{3}+\ell \tau_{0}, t_{3}+(\ell+1) \tau_{0}\right)$ to ensure that the uniform analyticity radius of $\eta(\cdot, t)$ is bounded below by $\delta\left(\tau_{0}\right)$ for all $t \in\left[t_{3}+(\ell+1) \tau_{0}, t_{3}+(\ell+2) \tau_{0}\right)$. Since this holds for all $\ell \geq 0$, this shows that the real analyticity radius of $\eta(\cdot, t)$ is bounded below by $\delta\left(\tau_{0}\right)$ for all $t \geq t_{0}$, where $t_{0}:=t_{3}+\tau_{0}$.

\section{Proof of Theorem 2}

We translate the results from the previous section into the setting of Gevrey norms. To this end, let us first define the analytic Gevrey class, $G^{1}\left(\mathbb{T}^{d}\right)$, as the class of functions, $g$, for which there exists constants $M, \lambda>0$ such that

$$
\left\|\partial^{\beta} g\right\|_{L^{\infty}} \leq M \frac{\beta !}{\lambda^{|\beta|}}, \quad \beta \in \mathbb{N}_{0}^{d}, \quad x \in \mathbb{T}^{d} .
$$


Let us also define the analytic Gevrey norm in the functional setting described in Section 2.1 by

$$
\|g\|_{\alpha, \lambda}:=\left\|A^{\alpha / 2} e^{\lambda A^{1 / 2}} g\right\|_{L^{2}} .
$$

We will make use of the following result, which characterizes $G^{1}\left(\mathbb{T}^{d}\right)$ in terms of the Gevrey norm. It can be found in greater generality in [36]. Since we are concerned with the dependencies of the constants, we supply a proof here that uses part of the proof of the more general result in [36] as a black box (see also [37]).

Lemma 5.1. If $g \in G^{1}\left(\mathbb{T}^{d}\right)$ for some $M, \lambda>0$, then for all $\gamma<\lambda$, there exists an absolute constant $C_{0}>0$, independent of $g, \gamma, \lambda$, such that

$$
\left\|e^{\gamma A^{1 / 2}} g\right\|_{L^{2}} \leq C_{0} M .
$$

Proof. Observe that

$$
\left\|\nabla^{\beta} g\right\|_{L^{2}} \leq(2 \pi)^{d}\left\|\nabla^{\beta} g\right\|_{L^{\infty}} \leq(2 \pi)^{d} M \frac{\beta !}{\lambda^{|\beta|}}, \quad \beta \in \mathbb{N}_{0}^{d},
$$

It follows from the proof of Theorem 4 in [36] that

$$
\left\|e^{\gamma A^{1 / 2}} g\right\|_{L^{2}} \leq C M
$$

for some absolute constant $C>0$, independent of $g, \gamma, \lambda$.

Combining Lemma 5.1 with Corollaries 4.1.1 and 4.1.1.1, we have the following bounds for the Gevrey norms of the reference solution, $u$, and the data assimilation solution, $v$.

Corollary 5.1.1. Let $f$ satisfy (4.1) and $\int_{\mathbb{T}^{2}} f d x=0$. Let $u_{0} \in V$ and $\nabla \times v_{0} \in L^{\infty}\left(\mathbb{T}^{2}\right)$ such that $\int_{\mathbb{T}^{2}} v_{0} d x=0$. Then there exists an absolute constant $c_{1}>0$ such that for $\mu$ sufficiently large and $m$ satisfying

$$
2^{m} \geq c_{1} \mu\left(1+\log _{+} \widetilde{\mathcal{G}}_{\infty}\right)^{2}
$$

there exists $t_{0}>0$ and absolute constants $C_{0}, C_{1}>0$ such that

$$
\begin{aligned}
& \sup _{t \geq t_{0}}\left\|e^{\gamma A^{1 / 2}} u(\cdot, t)\right\|_{V} \leq C_{0} \mathcal{G}_{\infty}, \quad \gamma<\delta_{0}:=\min \left\{\delta_{0}\left(t_{*}\right), \delta_{f}\right\}, \\
& \sup _{t \geq t_{0}}\left\|e^{\gamma A^{1 / 2}} v(\cdot, t)\right\|_{V} \leq C_{1} \frac{\widetilde{\mathcal{G}}_{\infty}}{\mu}, \quad \gamma<\delta_{*}:=\min \left\{\frac{\widetilde{\mathcal{G}}_{\infty}}{c_{2} \mathcal{G}_{\infty} \mu^{3 / 2}}, \delta_{0}, \delta_{f}\right\},
\end{aligned}
$$

where $\delta_{0}(t)$ is given in Lemma 4.1 .1 and

$$
t_{*}:=\frac{1}{K_{0}\left(1+\log _{+} \mathcal{G}_{\infty}\right)^{1 / 2}} \min \left\{\frac{\mathcal{G}_{\infty}^{4}}{M_{f}^{2}\left(1+\log _{+} \mathcal{G}_{\infty}\right)^{1 / 2}}, \frac{1}{\mathcal{G}_{\infty}}\right\} .
$$

Using the elementary fact that $|x| e^{-a|x|} \leq \mathrm{Ca}^{-1}$, we immediately have the following.

Corollary 5.1.2. Under the hypotheses of Corollary 5.1.1, there exists $t_{0}>0$ and an absolute constant $C_{0}^{\prime}>0$ such that

$$
\sup _{t \geq t_{0}}\left\|e^{\gamma A^{1 / 2}} A u(\cdot, t)\right\|_{H} \leq C_{0}^{\prime} \frac{\mathcal{G}_{\infty}}{\delta_{0}}, \quad \gamma<\lambda .
$$


We are now ready to prove Theorem 2 .

Proof of Theorem 2. Let $\overline{\mathcal{G}}_{\infty}:=\widetilde{\mathcal{G}}_{\infty}+\mathcal{G}_{\infty}$. Suppose that

$$
\mu \geq c_{0} \max \left\{\overline{\mathcal{G}}_{\infty}^{2}\left(1+\log _{+} \overline{\mathcal{G}}_{\infty}\right)^{2}, \frac{M_{f}^{2 / 3} \widetilde{\mathcal{G}}_{\infty}^{2 / 3}}{\mathcal{G}_{\infty}^{2}}\left(1+\log _{+} \widetilde{\mathcal{G}}_{\infty}\right)^{1 / 3},\left(1+\log _{+} \widetilde{\mathcal{G}}_{\infty}\right)^{3}, \frac{M_{F}}{\mathcal{G}_{\infty}}, \frac{\widetilde{\mathcal{G}}_{\infty}}{\mathcal{G}_{\infty}}, 1\right\}
$$

and that $m$ satisfies $2^{m} \geq c_{1} \mu\left(1+\log _{+} \widetilde{\mathcal{G}}_{\infty}\right)^{2}$, where $c_{0}, c_{1}$ are given as in Proposition 4.1 , but possibly larger (see the estimates performed below). Observe that

$$
\overline{\mathcal{G}}_{\infty}^{2}\left(1+\log _{+} \overline{\mathcal{G}}_{\infty}\right)^{2} \geq \frac{\widetilde{\mathcal{G}}_{\infty}^{2 / 3}}{\mathcal{G}_{\infty}^{2 / 3}}\left(1+\log _{+} \widetilde{\mathcal{G}}_{\infty}\right)^{1 / 3}
$$

so that we may apply Proposition 4.1. Let $t_{0}$ be given as in Proposition 4.1. Let $\delta_{0}$ and $\delta_{*}$ be given by (5.3), (5.4), respectively. Then Corollary 5.1.1 and 5.1.2 imply that there exist absolute constants $C_{0}, C_{1}>0$, independent of $u, v$, such that

$$
\sup _{t \geq t_{0}}\left\|e^{\gamma A^{1 / 2}} u(\cdot, t)\right\|_{V} \leq C_{0} \mathcal{G}_{\infty}, \quad \sup _{t \geq t_{0}}\left\|e^{\gamma A^{1 / 2}} A u(\cdot, t)\right\|_{H} \leq C_{0}^{\prime} \frac{\mathcal{G}_{\infty}}{\delta_{0}}, \quad \gamma<\delta_{0},
$$

and

$$
\sup _{t \geq t_{0}}\left\|e^{\gamma A^{1 / 2}} v(\cdot, t)\right\|_{V} \leq C_{1} \widetilde{\mathcal{G}}_{\infty}, \quad \gamma<\delta_{*}
$$

Fix $\gamma<\delta_{*}$. We consider the equation for the difference $\mathbf{w}=v-u$ :

$$
\frac{d \mathbf{w}}{d t}+\nu A \mathbf{w}+B(u, \mathbf{w})+B(\mathbf{w}, u)+B(\mathbf{w}, \mathbf{w})=-\mu P_{2^{m}} \mathbf{w}, \quad \mathbf{w}_{0}(x)=\mathbf{w}\left(x, t_{0}\right) .
$$

Let us take the $L^{2}$ inner product of (5.8) with $A e^{\gamma A^{1 / 2}} \mathbf{w}$ and integrate by parts to obtain

$$
\begin{aligned}
& \frac{1}{2} \frac{d}{d t}\left\|e^{\gamma A^{1 / 2}} \mathbf{w}\right\|_{V}^{2}+\left\|A e^{\gamma A^{1 / 2}} \mathbf{w}\right\|_{H}^{2}=-\left\langle A^{1 / 2} e^{\gamma A^{1 / 2}} P_{2^{m}} \mathbf{w}, A^{1 / 2} e^{\gamma A^{1 / 2}} \mathbf{w}\right\rangle \\
& -\left\langle A^{1 / 2} e^{\gamma A^{1 / 2}} B(u, \mathbf{w}), A^{1 / 2} e^{\gamma A^{1 / 2}} \mathbf{w}\right\rangle-\left\langle A^{1 / 2} e^{\gamma A^{1 / 2}} B(\mathbf{w}, u), A^{1 / 2} e^{\gamma A^{1 / 2}} \mathbf{w}\right\rangle \\
& -\left\langle A^{1 / 2} e^{\gamma A^{1 / 2}} B(\mathbf{w}, \mathbf{w}), A^{1 / 2} e^{\gamma A^{1 / 2}} \mathbf{w}\right\rangle \\
& =I+I I+I I I+I V .
\end{aligned}
$$

For $I$, first observe that

$$
-\mu P_{2^{m}} \delta=\mu\left(\delta-P_{2^{m}} \delta\right)-\mu \delta .
$$


Let $Q_{m}=I-P_{2^{m}}$. By Plancherel's theorem, the Cauchy-Schwarz inequality, Poincaré inequality, Young's inequality, and assumption on $\mu$, it follows that

$$
\begin{aligned}
\mu\left|\left\langle S_{m} \mathbf{w}, A e^{2 \gamma A^{1 / 2}} \mathbf{w}\right\rangle\right| & \leq \mu\left\|Q_{m} e^{\gamma A^{1 / 2}} \mathbf{w}\right\|_{H}\left\|A e^{\gamma A^{1 / 2}} \mathbf{w}\right\|_{H}-\mu\left\|e^{\lambda A^{1 / 2}} \mathbf{w}\right\|_{V}^{2} \\
& \leq C \frac{\mu}{2^{m}}\left\|e^{\gamma A^{1 / 2}} \mathbf{w}\right\|_{V}\left\|A e^{\gamma A^{1 / 2}} \mathbf{w}\right\|_{H}-\mu\left\|e^{\lambda A^{1 / 2}} \mathbf{w}\right\|_{V}^{2} \\
& \leq-\mu\left(1-\frac{2 \mu}{2^{2 m}}\right)\left\|e^{\gamma A^{1 / 2}} \mathbf{w}\right\|_{V}^{2}+\frac{1}{8}\left\|A e^{\gamma A^{1 / 2}} \mathbf{w}\right\|_{H}^{2} \\
& \leq-\frac{3 \mu}{4}\left\|e^{\gamma A^{1 / 2}} \mathbf{w}\right\|_{V}^{2}+\frac{1}{8}\left|A e^{\gamma A^{1 / 2}} v\right|^{2} .
\end{aligned}
$$

Now let us treat $I I, I I I, I V$. Denote by $\mathcal{L}(\mathbf{w})$ the quantity

$$
\mathcal{L}(\mathbf{w}):=1+\log \left(\frac{\left\|A e^{\gamma A^{1 / 2}} \mathbf{w}\right\|_{H}^{2}}{\left\|e^{\gamma A^{1 / 2}} \mathbf{w}\right\|_{V}^{2}}\right) .
$$

Then using Plancherel's theorem, the fact that $e^{\gamma|k|} \leq e^{\gamma|k-\ell|} e^{\gamma|\ell|}$, then applying the CauchySchwarz inequality, Lemma 2.2.3 (Agmon's inequality), (5.6), and (5.5) we have

$$
\begin{aligned}
\left|\left\langle e^{\gamma A^{1 / 2}} B(u, \mathbf{w}), A e^{\gamma A^{1 / 2}} \mathbf{w}\right\rangle\right| & \leq\left\|e^{\gamma A^{1 / 2}} u\right\|_{\mathcal{W}}\left\|e^{\gamma A^{1 / 2}} \mathbf{w}\right\|\left|A e^{\gamma A^{1 / 2}} \mathbf{w}\right| \\
& \leq C \frac{\mathcal{G}_{\infty}^{2}}{\delta_{0}}\left\|e^{\lambda A^{1 / 2}} \mathbf{w}\right\|^{2}+\frac{1}{8}\left|A e^{\lambda A^{1 / 2}} \mathbf{w}\right|^{2} \\
& \leq \frac{\mu}{8}\left\|e^{\lambda A^{1 / 2}} \mathbf{w}\right\|^{2}+\frac{1}{8}\left\|A e^{\lambda A^{1 / 2}} \mathbf{w}\right\|_{H}^{2},
\end{aligned}
$$

For $I I I$, we estimate exactly as in $I I$, but apply Lemma 2.2.4 (Brezis-Gallouet inequality) instead of Agmon's inequality, and use (5.6) to obtain

$$
\begin{aligned}
\left|\left\langle e^{\gamma A^{1 / 2}} B(\mathbf{w}, u), A e^{\gamma A^{1 / 2}} \mathbf{w}\right\rangle\right| & \leq\left\|e^{\gamma A^{1 / 2}} \mathbf{w}\right\|_{V}\left(1+\log \left(\frac{\left\|A e^{\gamma A^{1 / 2}} \mathbf{w}\right\|_{H}^{2}}{\left\|e^{\gamma A^{1 / 2}} \mathbf{w}\right\|_{V}^{2}}\right)\right)\left\|e^{\gamma A^{1 / 2}} u\right\|_{V}\left\|A e^{\gamma A^{1 / 2}} \mathbf{w}\right\|_{H} \\
& \leq C \mathcal{L}(\mathbf{w})\left\|e^{\gamma A^{1 / 2}} u\right\|_{V}^{2}\left\|e^{\gamma A^{1 / 2}} \mathbf{w}\right\|_{V}^{2}+\frac{1}{8}\left\|A e^{\gamma A^{1 / 2}} \mathbf{w}\right\|_{H}^{2} \\
& \leq C \mathcal{L}(\mathbf{w}) \mathcal{G}_{\infty}^{2}\left\|e^{\gamma A^{1 / 2}} \mathbf{w}\right\|_{V}^{2}+\frac{1}{8}\left\|A e^{\gamma A^{1 / 2}} \mathbf{w}\right\|_{H}^{2}
\end{aligned}
$$

Similar to $I I I$, we estimate $I V$ as

$$
\begin{aligned}
\left|\left\langle e^{\gamma A^{1 / 2}} B(\mathbf{w}, \mathbf{w}), A e^{\gamma A^{1 / 2}} \mathbf{w}\right\rangle\right| & \leq C\left\|e^{\gamma A^{1 / 2}} \mathbf{w}\right\|_{V}^{4} \mathcal{L}(\mathbf{w})+\frac{1}{8}\left\|A e^{\gamma A^{1 / 2}} \mathbf{w}\right\|_{H}^{2} \\
& \leq C \overline{\mathcal{G}}_{\infty}^{2}\left\|e^{\gamma A^{1 / 2}} \mathbf{w}\right\|_{V}^{2} \mathcal{L}(\mathbf{w})+\frac{1}{8}\left\|A e^{\gamma A^{1 / 2}} \mathbf{w}\right\|_{H}^{2} .
\end{aligned}
$$

Thus, combining (5.11), and (5.13)-(5.15), we arrive at

$$
\frac{d}{d t}\left\|e^{\gamma A^{1 / 2}} \mathbf{w}\right\|_{V}^{2}+\frac{5}{4} \mu\left\|e^{\gamma A^{1 / 2}} \mathbf{w}\right\|_{V}^{2}+\left\|A e^{\gamma A^{1 / 2}} \mathbf{w}\right\|_{H}^{2} \leq C \overline{\mathcal{G}}_{\infty}^{2}\left\|e^{\gamma A^{1 / 2}} \mathbf{w}\right\|_{V}^{2} \mathcal{L}(\mathbf{w})
$$


In particular, we have

$$
\begin{aligned}
\frac{d}{d t}\left\|e^{\gamma A^{1 / 2}} \mathbf{w}\right\|_{V}^{2}+\frac{5}{4} \mu\left\|e^{\gamma A^{1 / 2}} \mathbf{w}\right\|_{V}^{2} & \leq\left\|e^{\gamma A^{1 / 2}} \mathbf{w}\right\|_{V}^{2}\left(C \overline{\mathcal{G}}_{\infty}^{2} \mathcal{L}(\mathbf{w})-\frac{\left\|A e^{\gamma A^{1 / 2}} \mathbf{w}\right\|_{H}^{2}}{\left\|e^{\gamma A^{1 / 2}} \mathbf{w}\right\|_{V}^{2}}\right) \\
& \leq C \overline{\mathcal{G}}_{\infty}^{2}\left(1+\log \left(C \overline{\mathcal{G}}_{\infty}^{2}\right)\right)\left\|e^{\gamma A^{1 / 2}} \mathbf{w}\right\|^{2},
\end{aligned}
$$

where we used the fact that $a \log x-x \leq a \log a$. Therefore, by (5.5), we obtain

$$
\frac{d}{d t}\left\|e^{\gamma A^{1 / 2}} \mathbf{w}\right\|^{2}+\mu\left\|e^{\gamma A^{1 / 2}} \mathbf{w}\right\|^{2} \leq 0
$$

An application of Gronwall's inequality over the interval $\left[t_{0}, \infty\right)$ implies that $\left\|e^{\gamma A^{1 / 2}} \mathbf{w}\right\|^{2}$ converges to 0 exponentially, for all $\gamma<\delta_{*}$.

Acknowledgments. The authors would like to thank Edriss S. Titi for insightful discussion in the course of this work. A. Biswas was partially supported by the NSF grant DMS 1517027.

\section{REFERENCES}

[1] V. Bjerknes. Das Problem der Wettervorhersage, betrachtet vom Standpunkte der Mechanic und der Physik. Meteorol. Z., pages 1-7, 1904.

[2] J. Charney. Dynamical forecasting by numerical process. Compendium of Meteorology, pages 470-482, 1951.

[3] A. Azouani, E. Olson, and E.S. Titi. Continuous data assimilation using general interpolant observables. J. Nonlinear Sci., 24:277-304, 2014.

[4] D.A.F Albanez, H.J. Nessenzveig Lopes, and E.S. Titi. Continuous data assimilation for the threedimensional Navier-Stokes- $\alpha$ model. Asymptot. Anal., 97:139-164, 2016.

[5] A. Azouani and E.S. Titi. Feedback control of nonlinear dissipative systems by finite determining parameters-a reaction-diffusion paradigm. Evol. Equ. Control Theory, 4:579-594, 2014.

[6] A. Farhat, M.S. Jolly, and E.S. Titi. Continuous data assimilation for the 2D bénard convection through velocity measurements alone. Phys. D, 303:59-66, 2015.

[7] A. Farhat, E. Lunasin, and E.S. Titi. Abridged continuous data assimilation for the 2D Navier-Stokes equations utilizing measurements of only one component of the velocity field. J. Math. Fluid Mech., 18(1):123, 2016.

[8] A. Farhat, E. Lunasin, and E.S. Titi. Data assimilation for 3D bénard convection in porous media employing only temperature measurements. J. Math. Anal. Appl., 438(1):492-506, 2016.

[9] P.A. Markowich, E.S. Titi, and S. Trabelsi. Continuous data assimilation for the three-dimensional Brinkman-Forchheimer-extended Darcy model. Nonlinearity, 29(4):1292-1328, 2016.

[10] H. Bessaih, E. Olson, and E.S. Titi. Continuous data assimilation with stochastically noisy data. Nonlinearity, 28(3):729-753, 2015.

[11] C. Foias, C. Mondaini, and E.S. Titi. A discrete data assimilation scheme for the solutions of the 2D Navier-Stokes equations and their statistics. arXiv.1602.059999v1, pages 1-31, 18 Feb 2016.

[12] C. Foias and G. Prodi. Sur le comportement global des solutions non stationnaires de èquations de NavierStokes en dimension deux. Rend. Sem. Mat. Univ. Padov., 39:1-34, 1967.

[13] C. Foias and R. Temam. Determination of the solutions of the Navier-Stokes equations by a set of nodal values. Math. Comp., 43(167):117-133, 1984.

[14] D.A. Jones and E.S. Titi. On the number of determining nodes for the 2D Navier-Stokes equations. J. Math. Anal. Appl., 168(1):72-88, 1992.

[15] D.A. Jones and E.S. Titi. Determining finite volume elements for the 2D Navier-Stokes equations: Experimental mathematics: computational issues in nonlinear science. Phys. D, 60(1-4):165-174, 1992.

[16] D.A. Jones and E.S. Titi. Upper bounds on the number of determining modes, nodes, and volume elements for the Navier-Stokes equations. Indiana Univ. Math. J., 42(3):875-887, 1993. 
[17] W. von Wahl. Estimating $\nabla u$ by div $u$ and curlu. Math. Methods Appl. Sci., 15(2):123-143, 1992.

[18] E. Olson. private communication. 2016.

[19] M. Gesho, E. Olson, and E.S. Titi. A computational study of a data assimilation algorithm for the twodimensional Navier-Stokes equations. Commun. Comput. Phys., 19(4):1094-1110, 2016.

[20] I. Kukavica. On the dissipative scale for the Navier-Stokes equation. Indiana Univ. Math. J., 48(3):10571081, 1999.

[21] Y. Meyer. Oscillating patterns in some nonlinear evolution equations. Mathematical foundation of turbulent viscous flows, 1871:101-187, 2006.

[22] J.T. Workman. End-point estimates and multi-parameter paraproducts on higher dimensional tori. Rev. Mat. Iberoam., 26(2):591-610, 2010.

[23] T. Kato and G. Ponce. Commutator estimates and the Euler and Navier-Stokes equation. Comm. Pure. Appl. Math., 41(7):891-907, 1988.

[24] C. Kenig, G. Ponce, and L. Vega. Well-posedness of the initial value problem for the korteweg-de-vries. Comm. Pure. Appl. Math., 41(7):891-907, 1988.

[25] R. Danchin. Fourier Methods in PDEs. Lecture notes, pages 1-91, 2005.

[26] H. Bahouri, J-Y. Chemin, and R. Danchin. Fourier analysis and nonlinear partial differential equations. Grundlehren der Mathematischen Wissenschaften [Fundamental Principles of Mathematical Sciences]. Springer, Heidelberg, 2011.

[27] L. Grafakos. Classical Harmonic Analysis, Third Edition. Springer Graduate Texts in Mathematics 249. Springer, New York, 2008.

[28] L. Grafakos. Modern Harmonic Analysis, Third Edition. Springer Graduate Texts in Mathematics 250. Springer, New York, 2008.

[29] P. Constantin and C. Foias. Navier-Stokes equations. Chicago Lectures in Mathematics. University of Chicago Press, Chicago, IL, 1988.

[30] C. Foias, O.P. Manley, R. Rosa, and R. Temam. Navier-Stokes equations and turbulence. Encyclopedia of Mathematics and its Applications. Cambridge University Press, Cambridge, 2001.

[31] R. Temam. Navier-Stokes equations: theory and numerical analysis. Studies in Mathematics and its Applications. North-Holland Publishing Co, Amsterdam-New York, 1977.

[32] R. Dascaliuc, C. Foias, and M.S. Jolly. Relations between energy and enstrophy on the global attractor of the 2-D Navier-Stokes equations. J. Dynam. Differential Equations, 17(4):643-736, 2005.

[33] E.M. Stein. Singular integrals and differentiability properties of functions. Princeton Mathematical Series, No. 30. Princeton University Press, Princeton, N.J., 1970.

[34] Z. Grujić and I. Kukavica. Space analyticity for the Navier-Stokes and related equations with initial data in $l^{p}$. J. Funct. Anal., 152(2):447-466, 1998.

[35] A.A. Ilyin and E.S. Titi. On the domain of analyticity and small scales for the solutions of the damped-driven 2D Navier-Stokes equations. Dyn. Partial Differ. Equ., 4(2):111-127, 2007.

[36] C.D. Levermore and M. Oliver. Analyicity of solutions for a generalized Euler equation. J. Differential Equations, 133(2):321-339, 1997.

[37] M. Oliver and E.S. Titi. Remark on the rate of decay of higher order derivatives for solutions to the Navier-Stokes equations. J. Funct. Anal., 172(1):1-18, 2000.

${ }^{1}$ Department of Mathematics, University of Maryland-Baltimore County, Baltimore, MD 21250

E-mail address, A. Biswas: abiswas@umbc.edu

2 Department of Mathematics, Tulane University, New Orleans, LA 70118

E-mail address, V. R. Martinez: vmartin6@tulane.edu $\dagger$ 